BULLETIN (New Series) OF THE

AMERICAN MATHEMATICAL SOCIETY

Volume 37, Number 3, Pages 209-249

S 0273-0979(00)00865-X

Article electronically published on April 5, 2000

\title{
EIGENVALUES, INVARIANT FACTORS, HIGHEST WEIGHTS, AND SCHUBERT CALCULUS
}

\author{
WILLIAM FULTON
}

\begin{abstract}
We describe recent work of Klyachko, Totaro, Knutson, and Tao that characterizes eigenvalues of sums of Hermitian matrices and decomposition of tensor products of representations of $G L_{n}(\mathbb{C})$. We explain related applications to invariant factors of products of matrices, intersections in Grassmann varieties, and singular values of sums and products of arbitrary matrices.
\end{abstract}

Recent breakthroughs, primarily by A. Klyachko, B. Totaro, A. Knutson, and T. Tao, with contributions by P. Belkale and C. Woodward, have led to complete solutions of several old problems involving the various notions in the title. Our aim here is to describe this work and especially to show how these solutions are derived from it. Along the way, we will see that these problems are also related to other areas of mathematics, including geometric invariant theory, symplectic geometry, and combinatorics. In addition, we present some related applications to singular values of arbitrary matrices.

Although many of the theorems we state here have not appeared elsewhere, their proofs are mostly "soft" algebra based on the hard geometric or combinatorial work of others. Indeed, this paper emphasizes concrete elementary arguments. We do give some new examples and counterexamples and raise some new open questions.

We have attempted to point to the sources and to some of the key partial results that had been conjectured or proved before. However, there is a very large literature, particularly for linear algebra problems about eigenvalues, singular values, and invariant factors. We have listed only a few of these articles, from whose bibliographies, we hope, an interested reader can trace the history; we apologize to the many whose work is not cited directly.

We begin in the first five sections by describing each of the problems, together with some of their early histories, and we state as theorems the new solutions to these problems. In Section 6 we describe the steps toward these solutions that were carried out before the recent breakthroughs. Then we discuss the recent solutions and explain how these theorems follow from the work of the above mathematicians. Sections 7, 8, 9, and 10 also contain variations and generalizations of some of the theorems stated in the first five sections, as well as attributions of the theorems to their authors.

One of our fascinations with this subject, even now that we have proofs of the theorems, is the challenge to understand in a deeper way why all these subjects are

Received by the editors in July 1999 and in revised form January 3, 2000.

2000 Mathematics Subject Classification. Primary 15A42, 22E46, 14M15; Secondary 05E15, 13F10, 14C17, 15A18, 47B07.

The author was partly supported by NSF Grant \#DMS9970435. 
so closely related. It is a particular challenge in each of these areas to understand why the solutions can be described inductively.

I am grateful to many people for advice and help in preparing this article, especially: P. Belkale, A. Buch, L. Chen, J. Day, P. Diaconis, A. Knutson, R. Lazarsfeld, C. K. Li, A. Okounkov, Y. T. Poon, P. Pragacz, J. F. Queiró, P. Sarnak, F. Sottile, R. Steinberg, J. Stembridge, T. Y. Tam, H. Tamvakis, T. Tao, B. Totaro, C. Woodward, A. Zelevinsky, and a referee.

\section{Contents}

1. Eigenvalues of sums of Hermitian and real symmetric matrices . . 210

2. Invariant factors . . . . . . . . . . . . . . . . . 217

3. Highest weights . . . . . . . . . . . . . . . . 220

4. Schubert calculus . . . . . . . . . . . . . . . . . . 223

5. Singular values of sums and products . . . . . . . . . . . . . 224

6. First steps toward the proofs . . . . . . . . . . . . . . . . 227

7. Filtered vector spaces, geometric invariant theory and stability . . . . . . . . . . . . . . . . . . . . . . . . 231

8. Coadjoint orbits and moment maps . . . . . . . . . . . . . . . 236

9. Saturation . . . . . . . . . . . . . . . . . . . 238

10. Proofs of the theorems . . . . . . . . . . . . . . . 239

11. Final remarks . . . . . . . . . . . . . . . . . . . . 245

\section{Eigenvalues of sums of Hermitian and Real Symmetric matrices}

The first problem goes back to the nineteenth century: What can be said about the eigenvalues of a sum of two Hermitian (or real symmetric) matrices, in terms of the eigenvalues of the summands?

It is a basic fact of linear algebra that all of the eigenvalues of any Hermitian or real symmetric matrix are real. We consider $n$ by $n$ matrices, with $n$ fixed. If $A$ is a real symmetric matrix, its eigenvalues describe the quadratic form $q_{A}(x)=x^{t} A x$ in an appropriate orthogonal coordinate system. For example, if the eigenvalues are positive, the inverses of the square roots of the eigenvalues are half the lengths of the principal axes of the ellipsoid $q_{A}(x)=1$.

We always list the $n$ eigenvalues of such a matrix in decreasing order, including any eigenvalue as often as its multiplicity. If $A, B$, and $C$ are Hermitian $n$ by $n$ matrices, we denote the eigenvalues of $A$ by

$$
\alpha: \quad \alpha_{1} \geq \alpha_{2} \geq \ldots \geq \alpha_{n},
$$

and similarly write $\beta$ and $\gamma$ for the eigenvalues (spectra) of $B$ and $C$. The question becomes:

What $\alpha, \beta$, and $\gamma$ can be the eigenvalues of $n$ by $n$ Hermitian (or real symmetric) matrices $A, B$, and $C$, with $C=A+B$ ?

For example, one can fix $A$ and take $B$ small, thus regarding $C$ as a deformation of $A$. In the real symmetric case, one is then asking how the shape of the hypersurface $q_{C}(x)=1$ compares with that of $q_{A}(x)=1$.

One can, if one wishes, take $A=D(\alpha)$ to be the diagonal matrix with entries $\alpha_{1}, \ldots, \alpha_{n}$ down the diagonal, and $B=U D(\beta) U^{*}$, with $U$ a unitary (or orthogonal in the real symmetric case) matrix. We are looking for the eigenvalues of $D(\alpha)+$ 
$U D(\beta) U^{*}$ as $U$ varies over the unitary group $U(n)$. This was the approach in much of the classical work on the problem, but it will not play an important role here.

There is one obvious necessary condition, that the trace of $C$ be the sum of the traces of $A$ and $B$ :

$$
\sum_{i=1}^{n} \gamma_{i}=\sum_{i=1}^{n} \alpha_{i}+\sum_{i=1}^{n} \beta_{i}
$$

There is a long history of results that put additional necessary conditions on the possible eigenvalues. The first goes back more than a century and is a reasonable exercise for a linear algebra class:

$$
\gamma_{1} \leq \alpha_{1}+\beta_{1} .
$$

The first significant result was given in 1912 by H. Weyl [W]:

$$
\gamma_{i+j-1} \leq \alpha_{i}+\beta_{j} \text { whenever } i+j-1 \leq n .
$$

Here is a typical application of these inequalities. If $A$ and $B$ are Hermitian $n$ by $n$ matrices that differ by a matrix of rank at most $r$, then their eigenvalues $\alpha$ and $\beta$ satisfy the inequalities

$$
\alpha_{k+r} \leq \beta_{k} \text { and } \beta_{k+r} \leq \alpha_{k} \text { for } 1 \leq k, k+r \leq n .
$$

Indeed, these inequalities follow by applying (2) to the triples $(B, A-B, A)$ and $(A, B-A, B)$, with $j=r+1$. When $r=1$, this is an interlacing theorem: between any two odd numbered (or even numbered) eigenvalues of $A$ there is at least one eigenvalue of $B$. There are similar results for eigenvalues of principal minors of $A$, which we describe in Section 6.

In fact, for $n=2$, it is not hard to verify directly that the conditions $(*),(1)$, and (2), which say

$$
\begin{aligned}
& \gamma_{1}+\gamma_{2}=\alpha_{1}+\alpha_{2}+\beta_{1}+\beta_{2} \\
& \gamma_{1} \leq \alpha_{1}+\beta_{1}, \quad \gamma_{2} \leq \alpha_{2}+\beta_{1}, \text { and } \gamma_{2} \leq \alpha_{1}+\beta_{2}
\end{aligned}
$$

are both necessary and sufficient for the existence of 2 by 2 Hermitian (or real symmetric) matrices with these eigenvalues.

In 1949 K. Fan $[\mathrm{F}$ ) found some other necessary conditions:

$$
\sum_{i=1}^{r} \gamma_{i} \leq \sum_{i=1}^{r} \alpha_{i}+\sum_{i=1}^{r} \beta_{i} \quad \text { for any } r<n .
$$

This question was featured in Gel'fand's seminar in Moscow. In 1950 V. B. Lidskii [L1], cf. [BG], found the following necessary condition. For this, regard an $n$-tuple $\alpha$ of eigenvalues as a point $\left(\alpha_{1}, \ldots, \alpha_{n}\right)$ in $\mathbb{R}^{n}$. This result asserts that the point $\gamma$ must be in the convex hull of the points $\alpha+\beta_{\sigma}$, where $\sigma$ varies over the symmetric group $S_{n}$, and $\beta_{\sigma}$ denotes $\left(\beta_{\sigma(1)}, \ldots, \beta_{\sigma(n)}\right)$. Although this looks quite different from (1) - (3), H. Wielandt [Wi] showed that this geometric condition is equivalent to the inequalities:

$$
\sum_{i \in I} \gamma_{i} \leq \sum_{i \in I} \alpha_{i}+\sum_{i=1}^{r} \beta_{i}
$$

for every subset $I$ of $\{1, \ldots, n\}$ of cardinality $r$, for all $r<n$. Of course, the same inequalities are valid when the roles of $\alpha$ and $\beta$ are interchanged, and we include 
them in the list we are making. In 1962, A. Horn [H2] showed that the inequalities listed so far, with the addition of

$$
\gamma_{2}+\gamma_{3} \leq \alpha_{1}+\alpha_{3}+\beta_{1}+\beta_{3},
$$

are necessary and sufficient for the existence of 3 by 3 Hermitian matrices with these eigenvalues.

Other inequalities were found, all having the form

$\left(*_{I J K}\right)$

$$
\sum_{k \in K} \gamma_{k} \leq \sum_{i \in I} \alpha_{i}+\sum_{j \in J} \beta_{j}
$$

for certain subsets $I, J, K$ of $\{1, \ldots, n\}$ of the same cardinality $r$, with $r<n$. We always write the subsets in increasing order, so

$$
I=\left\{i_{1}<i_{2}<\ldots<i_{r}\right\}
$$

$J=\left\{j_{1}<\ldots<j_{r}\right\}$, and $K=\left\{k_{1}<\ldots<k_{r}\right\}$.

For example, there is a result of L. Freede and R. C. Thompson TF, which generalizes the Weyl, Fan, and Lidskii-Wielandt inequalities:

$$
\sum_{k \in K} \gamma_{k} \leq \sum_{i \in I} \alpha_{i}+\sum_{j \in J} \beta_{j} \text {, if } k_{p}=i_{p}+j_{p}-p \text { for } 1 \leq p \leq r .
$$

One has these inequalities for any $I$ and $J$, provided that $i_{r}+j_{r} \leq n+r$.

Note that any inequality of the form $\left(*_{I J K}\right)$ can be subtracted from the equality $(*)$ to give an inequality

$$
\sum_{k \in K^{c}} \gamma_{k} \geq \sum_{i \in I^{c}} \alpha_{i}+\sum_{j \in J^{c}} \beta_{j},
$$

where $I^{c}, J^{c}$, and $K^{c}$ are the complements of $I, J$, and $K$ in $\{1, \ldots, n\}$. We do not list any of these.

In his remarkable paper [H2], Horn undertook a systematic study of such inequalities. In fact, he prescribed sets of triples $(I, J, K)$, and he conjectured that the inequalities $\left(*_{I J K}\right)$ for these triples would give both necessary and sufficient conditions for a triple $(\alpha, \beta, \gamma)$ to arise as eigenvalues of Hermitian matrices $A, B$, and $C$ with $C=A+B$.

Horn defined sets $T_{r}^{n}$ of triples $(I, J, K)$ of subsets of $\{1, \ldots, n\}$ of the same cardinality $r$, by the following inductive procedure. Set

$$
U_{r}^{n}=\left\{(I, J, K) \mid \sum_{i \in I} i+\sum_{j \in J} j=\sum_{k \in K} k+r(r+1) / 2\right\} .
$$

All the triples that we have listed are in $U_{r}^{n}$. When $r=1$, set $T_{1}^{n}=U_{1}^{n}$. (The inequalities specified by $(I, J, K)$ in $T_{1}^{n}$ are Weyl's inequalities (2).) In general,

$$
\begin{gathered}
T_{r}^{n}=\left\{(I, J, K) \in U_{r}^{n} \mid \text { for all } p<r \text { and all }(F, G, H) \text { in } T_{p}^{r},\right. \\
\left.\sum_{f \in F} i_{f}+\sum_{g \in G} j_{g} \leq \sum_{h \in H} k_{h}+p(p+1) / 2\right\} .
\end{gathered}
$$

Horn's Conjecture. A triple $(\alpha, \beta, \gamma)$ occurs as eigenvalues of Hermitian $n$ by $n$ matrices $A, B, C$ with $C=A+B$ if and only if $\sum \gamma_{i}=\sum \alpha_{i}+\sum \beta_{i}$ and the inequalities $\left(*_{I J K}\right)$ hold for every $(I, J, K)$ in $T_{r}^{n}$, for all $r<n$.

B.V. Lidskii, son of V. B. Lidskii, announced a proof of Horn's conjecture in $1982[\mathbf{L} 2$, but no details of this have ever appeared. 
Theorem 1. Horn's conjecture is true.

In fact, from the recent work to be discussed below, the surprising inductive form of this eigenvalue problem can be given in another way. To state this we use a standard correspondence between finite sets $I=\left\{i_{1}<i_{2}<\ldots<i_{r}\right\}$ of $r$ positive integers, and partitions $\lambda=\left(\lambda_{1} \geq \lambda_{2} \geq \ldots \geq \lambda_{r}\right)$ of nonnegative integers of length at most $r$. This correspondence is obtained by defining

$$
\lambda=\lambda(I)=\left(i_{r}-r, i_{r-1}-(r-1), \ldots, i_{2}-2, i_{1}-1\right) .
$$

We let $J$ correspond to $\mu$, and $K$ correspond to $\nu$, by this same recipe. When we say $(\lambda, \mu, \nu)$ corresponds to $(I, J, K)$, we mean $(\lambda, \mu, \nu)=(\lambda(I), \lambda(J), \lambda(K))$. (To recover the subsets from the partitions, the integer $r$ must be specified, but this will always be the case for us.)

If $(I, J, K)$ corresponds to $(\lambda, \mu, \nu)$, note that $(I, J, K)$ is in $U_{r}^{n}$ exactly when $\sum \nu_{i}=\sum \lambda_{i}+\sum \mu_{i}$. Note also that each of $\lambda, \mu$, and $\nu$ is a sequence of $r$ real numbers listed in decreasing order, so it makes sense to ask if they arise as eigenvalues of a triple of Hermitian $r$ by $r$ matrices.

Theorem 2. A triple $(I, J, K)$ is in $T_{r}^{n}$ if and only if the corresponding triple $(\lambda, \mu, \nu)$ occurs as eigenvalues of a triple of Hermitian $r$ by $r$ matrices, with the third the sum of the first two.

For example, one can use this theorem to find (all) such triples with $r=n-1$. They consist of complements of integers $i, j$, and $k$ with $i+j=n+k$. The triples $(\lambda, \mu, \nu)$ corresponding to these subsets consist of partitions with only 1 's and 0 's, with $n-i, n-j$, and $n-k 1$ 's in $\lambda, \mu$, and $\nu$ respectively. These occur as eigenvalues of diagonal matrices of size $n-1$ with only 1's and 0's as entries; the first matrix has $n-i 1$ 's at the beginning of the diagonal, the second has $n-j 1$ 's at the end, and their sum has a total of $n-k 1$ 's at the ends. From (7) this gives the inequalities

$$
\gamma_{k} \geq \alpha_{i}+\beta_{j} \quad \text { if } \quad i+j=n+k .
$$

For another example, the triple $(I, J, K)=(\{1,3,5\},\{1,3,5\},\{2,4,6\})$ is in $T_{3}^{6}$. In this case the corresponding triple $(\lambda, \mu, \nu)=((2,1,0),(2,1,0),(3,2,1))$ arises from the triple of diagonal 3 by 3 matrices with diagonal entries $(2,1,0),(1,0,2)$, and $(3,1,2)$.

We will give several other characterizations of the sets $T_{r}^{n}$ in terms of LittlewoodRichardson coefficients (see Theorems 11 and 12) in Section 3.

Horn's paper also contains results and examples about the case of real symmetric matrices, but he did not explicitly make his conjecture for this case. In fact, some of the methods that were used to prove inequalities broke down in the real case (see Example 1), so there was some doubt whether the real symmetric and complex Hermitian cases would coincide; a positive answer, however, was conjectured in DST. Theorems 1 and 2 are indeed true in the real symmetric case:

Theorem 3. A triple $(\alpha, \beta, \gamma)$ occurs as eigenvalues for a triple of real symmetric matrices if and only if it occurs for a triple of complex Hermitian matrices.

There have also been a few cases of inequalites $\left(*_{I J K}\right)$ proved for Hermitian (selfconjugate) quaternionic matrices (e.g. $[\mathrm{X}]$ ). The complete answer, as suggested by Steinberg and Totaro, is the same as in the real and complex situations: 
Theorem 4. A triple $(\alpha, \beta, \gamma)$ occurs as eigenvalues for a triple of quaternionic Hermitian matrices if and only if it occurs for a triple of complex Hermitian matrices.

V. B. Lidskii and Horn investigated the situation when some inequality $\left(*_{I J K}\right)$ becomes an equality, and they stated and proved special cases of the following theorem.

Theorem 5. If Hermitian matrices $A, B$, and $C=A+B$ have eigenvalues $\alpha, \beta$, and $\gamma$, and some inequality $\left(*_{I J K}\right)$ occurs with equality for $(I, J, K)$ in $T_{r}^{n}$, then there is an $r$-dimensional subspace $L$ of $\mathbb{C}^{n}$ such that $A, B$, and $C$ map $L$ into itself.

This means that, after a unitary change of coordinates, the three matrices have a block diagonal form $\left(\begin{array}{cc}P & 0 \\ 0 & Q\end{array}\right)$ with $P r$ by $r$ and $Q n-r$ by $n-r$ Hermitian matrices.

Example 1. The assertion of Theorem 5 is not true for real symmetric matrices and real subspaces of $\mathbb{R}^{n}$, although it is true for $n \leq 5$. Here is an explicit example with $n=6$. Take $A$ to be any diagonal matrix with entries $(x, x, y, y, z, z)$ down the diagonal, with $x, y$, and $z$ distinct real numbers that sum to zero. Take $B$ to be the matrix

$$
\left(\begin{array}{cccccc}
15 & 0 & -32 & -3 & 35 & -3 \\
0 & 15 & 3 & -32 & 3 & 35 \\
-32 & 3 & -17 & 0 & 51 & 19 \\
-3 & -32 & 0 & -17 & -19 & 51 \\
35 & 3 & 51 & -19 & 2 & 0 \\
-3 & 35 & 19 & 51 & 0 & 2
\end{array}\right)
$$

which has eigenvalues $(56,56,28,28,-84,-84)$. Then $C=A+B$ will also have eigenvalues consisting of three pairs of distinct numbers that sum to zero. The inequality $\left(*_{I J K}\right)$, with $I=J=\{1,3,5\}$, and $K=\{2,4,6\}$ is an equality, since $\alpha_{1}+\alpha_{3}+\alpha_{5}=0, \beta_{1}+\beta_{3}+\beta_{5}=0$, and $\gamma_{2}+\gamma_{4}+\gamma_{6}=0$. There are exactly two 3 -dimensional subspaces preserved by $A$ and $B$ (and therefore $C$ ), and both are complex; letting $e_{1}, \ldots, e_{6}$ be the standard basis for $\mathbb{C}^{n}$, they are the span $L$ of $e_{1}+i e_{2}, e_{3}+i e_{5}$, and $e_{5}+i e_{6}$, and its orthogonal subspace $L^{\perp}$, spanned by $e_{1}-i e_{2}, e_{3}-i e_{5}$, and $e_{5}-i e_{6}$. (The fact that $C$ preserves these subspaces implies that its eigenvalues come in pairs as asserted.) We will see some explanation for this example later (cf. [TT2]).

It should be understood that one is looking for a minimal, or at least a small set of inequalities $\left(*_{I J K}\right)$. Any such inequality determines many others, and one usually does not want to list these trivial consequences. For example, from (5) one can deduce immediately that $\gamma_{2}+\gamma_{4} \leq \alpha_{1}+\alpha_{3}+\beta_{1}+\beta_{2}$, simply because $\gamma_{4} \leq \gamma_{3}$ and $\beta_{3} \leq \beta_{2}$. As A. Buch points out, there is a special situation for $n=2$, as the inequalities $\alpha_{1} \geq \alpha_{2}, \beta_{1} \geq \beta_{2}$, and $\gamma_{1} \geq \gamma_{2}$ follow from the equality (*) and the three inequalities $\left(*_{I J K}\right)$. With this exception, the inequalities in Horn's conjecture are in fact minimal for $n \leq 5$, which is the region that Horn investigated most thoroughly. We will see, however, that they are not minimal for larger $n$, although that was thought to be the case until quite recently.

Although the triple $(I, J, K)=(\{1,3,5\},\{1,3,5\},\{2,4,6\})$ is on Horn's list, as we have seen, the inequality

$$
\gamma_{2}+\gamma_{4}+\gamma_{6} \leq \alpha_{1}+\alpha_{3}+\alpha_{5}+\beta_{1}+\beta_{3}+\beta_{5}
$$


is redundant and follows in fact from $(*)$ and the fact that the eigenvalues are listed in decreasing order. Indeed, let $\alpha_{\mathrm{ev}}=\alpha_{2}+\alpha_{4}+\alpha_{6}$, and $\alpha_{\mathrm{od}}=\alpha_{1}+\alpha_{3}+\alpha_{5}$, and similarly for $\beta$ and $\gamma$. The fact that each of $\alpha, \beta$, and $\gamma$ is nonincreasing implies the inequalities $\alpha_{\mathrm{ev}} \leq \alpha_{\mathrm{od}}, \beta_{\mathrm{ev}} \leq \beta_{\mathrm{od}}$, and $\gamma_{\mathrm{ev}} \leq \gamma_{\mathrm{od}}$. But $(*)$ says that $\gamma_{\mathrm{od}}+\gamma_{\mathrm{ev}}=\alpha_{\mathrm{od}}+\alpha_{\mathrm{ev}}+\beta_{\mathrm{od}}+\beta_{\mathrm{ev}}$. Hence $\gamma_{\mathrm{ev}} \leq \alpha_{\mathrm{od}}+\beta_{\mathrm{od}}$, as asserted.

In fact, for $n=6$, this is the only triple on Horn's list that can be omitted (and the only triple that gives rise to an example like Example 1). As $n$ increases, however, the number of redundant triples on the list increases rapidly. Describing the actual minimal set, however, requires notions from other areas of mathematics and is postponed to later sections.

As this example indicates, one reason why the inequalities defining the realizable triples $(\alpha, \beta, \gamma)$ pose problems is because, together with equation $(*)$ and the inequalities $\left(*_{I J K}\right)$, one also has the $3 n-3$ inequalities $\alpha_{1} \geq \alpha_{2} \geq \ldots \geq \alpha_{n}$, $\beta_{1} \geq \beta_{2} \geq \ldots \geq \beta_{n}, \gamma_{1} \geq \gamma_{2} \geq \ldots \geq \gamma_{n}$.

There is also a literature describing the possibilities for a particular eigenvalue $\gamma_{k}$ (with $k$ fixed) of $C=A+B$ in terms of the eigenvalues of $A$ and B. In fact, this problem was solved much earlier (see Joh). The result is that the $k^{\text {th }}$ largest eigenvalue of $C$ can take on any value in an interval:

$$
\operatorname{Max}_{i+j=n+k} \alpha_{i}+\beta_{j} \leq \gamma_{k} \leq \operatorname{Min}_{i+j=k+1} \alpha_{i}+\beta_{j}
$$

We have seen in (2) and (11) that $\gamma_{k}$ satisfies these inequalities, and since the set in question is a projection of a convex set in $\mathbb{R}^{n}$ (or the image of the connected space $U(n)$ by a continuous map) it must be an interval. It therefore suffices to produce Hermitian matrices to achieve each of the endpoints of the displayed interval. Explicit diagonal matrices can be produced to achieve this [Joh]. This argument also shows that this question has the same answer for real symmetric matrices.

More generally, one can specify a subset $K$ of $\{1, \ldots, n\}$ and ask for the possible values of $\left\{\gamma_{k} \mid k \in K\right\}$, again with $\alpha$ and $\beta$ given. In principle, such results may be deduced from Theorem 1, although carrying this out does not appear to be easy. One always has the inequalities $\left(*_{F G H}\right)$ given for triples $(F, G, H)$, for $H$ a subset of $K$, and the duals $(7)$ of inequalities $\left(*_{F G H}\right)$ when $H$ contains the complement of $K$, together with the inequalities saying that the eigenvalues in $K$ form a weakly decreasing sequence. But already for $n=3$ and $K=\{1,3\}$ it is easy to see that such inequalities do not suffice: they do not imply that the missing $\gamma_{2}$, which is determined, is at least as large as $\gamma_{3}$.

Not all inequalities that have been found involving eigenvalues of sums of Hermitian matrices are linear. For example, M. Fiedler [Fi] showed that

$$
\operatorname{Min}_{\sigma \in S_{n}} \prod_{i=1}^{n}\left(\alpha_{i}+\beta_{\sigma(i)}\right) \leq \prod_{i=1}^{n} \gamma_{i} \leq \operatorname{Max}_{\sigma \in S_{n}} \prod_{i=1}^{n}\left(\alpha_{i}+\beta_{\sigma(i)}\right) .
$$

There are still some basic questions remaining. One of these is the following:

Which triples $(I, J, K)$ of subsets of cardinality $r$ in $\{1, \ldots, n\}$ give true inequalities $\left(*_{I J K}\right)$ for eigenvalues of all $n$ by $n$ Hermitian matrices $A, B, \mathrm{C}=\mathrm{A}+\mathrm{B}$ ?

Let us call this set $H_{r}^{n}$. This question was addressed by Horn [H2] and then by Zwahlen $[\mathrm{Zw}]$. Theorem 1 asserts that $T_{r}^{n} \subset H_{r}^{n}$, but how much larger is $H_{r}^{n}$ ? 
For $r \leq 2$, the answer is quite simple and was given by Horn and Zwahlen. The set $H_{1}^{n}$ consists of triples with $i_{1}+j_{1} \leq k_{1}+1$. The set $H_{2}^{n}$ consists of triples such that $i_{1}+j_{1} \leq k_{1}+1, i_{2}+j_{1} \leq k_{2}+1, i_{1}+j_{2} \leq k_{2}+1$, and $i_{1}+i_{2}+j_{1}+j_{2} \leq k_{1}+k_{2}+3$. In fact, they show that for $r \leq 2$ and any triple not satisfying these conditions, there are diagonal matrices $A, B$, and $C=A+B$ whose eigenvalues violate the inequality $\left(*_{I J K}\right)$. They prove some partial results for $r=3$. On the basis of the evidence in these papers, it was natural to hope that, if $(I, J, K)$ is given to be in $U_{r}^{n}$, then it is in $H_{r}^{n}$ only if it is in $T_{r}^{n}$, i.e., $H_{r}^{n} \cap U_{r}^{n}=T_{r}^{n}$. However, this is not true:

Example 2. Take $r=5, n=25$, and $I=J=\{1,3,4,16,21\}$ and $K=\{5,10,15$, $20,25\}$. This is in $H_{r}^{n} \cap U_{r}^{n}$. Indeed, it is easy to see, by the same argument as in the proof of (12), that for any weakly decreasing $n$-tuples $\alpha, \beta$, and $\gamma$ such that $\sum \gamma_{i}=\sum \alpha_{i}+\sum \beta_{i}$

$$
\begin{aligned}
\sum_{k \in K} \gamma_{k}=\sum_{s=0}^{4} \gamma_{5+5 s} & \leq \sum_{s=0}^{4} \alpha_{1+5 s}+\sum_{s=0}^{4} \beta_{1+5 s} \\
& \leq \sum_{i \in I} \alpha_{i}+\sum_{j \in J} \beta_{j} .
\end{aligned}
$$

To see that $(I, J, K)$ is not in $T_{5}^{25}$, one can use the triple $(F, G, H)$ in $T_{4}^{5}$, with $F=G=\{1,2,4,5\}$ and $H=\{2,3,4,5\}: \sum_{f \in F} i_{f}+\sum_{g \in G} j_{g}=82>80=$ $\sum_{h \in H} k_{h}+p(p+1) / 2$. Many other examples can be constructed by this method, but none of them are very small.

Zwahlen $[\mathrm{Zw}$ ] gave an example of a triple $(I, J, K)$ for which he produced violating matrices, but for which no diagonal matrices violate $\left(*_{I J K}\right)$; this was for $r=3, n=18, I=J=\{1,6,11\}, K=\{2,9,18\}$. Thompson and Therianos [TT1] gave the simpler triple $(\{1,3,5\},\{1,3,5\},\{2,3,6\})$ for $r=3, n=6$, with the same property. These triples, however, are not in $U_{r}^{n}$.

Example 3 (Buch). The triple $(I, J, K)=(\{1,3,5,6\},\{1,3,5,6\},\{2,3,6,9\})$ is in $U_{4}^{9} \backslash T_{4}^{9}$, but no diagonal 9 by 9 Hermitian matrices can have eigenvalues violating $\left(*_{I J K}\right)$. If $A$ is the diagonal matrix with diagonal entries $(2,0,1,2,0,1,0,0,0)$, and $B$ is the direct sum of the matrix $\left(\begin{array}{cc}1 / 2 & \sqrt{3} / 2 \\ \sqrt{3} / 2 & 3 / 2\end{array}\right)$ and the diagonal matrix with diagonal entries $(2,1,1,0,0,0,0)$, then the eigenvalues of $A, B$, and $C=A+B$ are $\alpha=\beta=(2,2,1,1,0,0,0,0,0)$, and $\gamma=(3,3,3,1,1,1,0,0,0)$. These violate $\left(*_{I J K}\right)$.

These examples indicate some of the subtlety of identifying triples that give correct inequalities and finding violating matrices for triples that do not.

It is interesting to note that much of the extreme behavior can be detected by matrices that are diagonal or close to diagonal, at least in low dimensions. Indeed, this must have been how many of the inequalities were discovered. However, we will see that the proofs of the theorems are almost opposite to this: the eigenvectors of the matrices produced by the proofs are in general position.

The theorems of this section give a clear picture of the set of all triples $(\alpha, \beta, \gamma)$ that occur as spectra of Hermitian $n$ by $n$ matrices $A, B$, and $C=A+B$. For small $n$ they give a reasonable set of inequalities that one can use to test if a particular triple $(\alpha, \beta, \gamma)$ occurs. For large $n$, however, the number of inequalities to be tested increases dramatically (even if decreased by using Theorem 13 below). When all of $\alpha, \beta$, and $\gamma$ are integral, one can test directly if $(\alpha, \beta, \gamma)$ occurs by using Theorem 11 below. When the eigenvalues are rational, one can multiply them all by a common 
denominator to reduce to the integral case. We do not know a similarly direct criterion for arbitrary real eigenvalues.

The necessary conditions of the theorems extend readily from the realm of finite dimensional Hermitian operators to that of compact self-adjoint operators on a Hilbert space. Indeed, it was in this context that Weyl [W] stated his results, cf. $\mathrm{Zw}$. Such an operator $A$ has a sequence of positive eigenvalues $\alpha_{1} \geq \alpha_{2} \geq \alpha_{3} \geq$ ... (each occurring according to multiplicity) and a similar sequence of negative eigenvalues. For simplicity we consider only the positive eigenvalues:

Theorem 6. Suppose $A, B$, and $C=A+B$ are compact self-adjoint operators on a Hilbert space, with $\alpha, \beta$, and $\gamma$ their sequences of positive eigenvalues, and assume that each of these sequences is infinite. Then $\left(*_{I J K}\right)$ holds for all $(I, J, K)$ in $T_{r}^{n}$, for all $n$ and $r$ with $r<n$.

The theorems here describe all possible eigenvalues of $A+B$ when $A$ and $B$ are $n$ by $n$ Hermitian matrices with eigenvalues $\alpha$ and $\beta$. In contrast, P. Biane Bi] has shown that, as $n$ gets large, for almost all choices of $A$ and $B$, the eigenvalues of $A+B$ are close to some $\gamma$ that depends only on $\alpha$ and $\beta$.

Much of the history of the eigenvalue problem before the recent events, together with an extensive bibliography, can be found in the survey [DST].

\section{INVARIANT FACTORS}

We turn now to quite a different problem. Consider an $n$ by $n$ matrix $A$ with entries in a discrete valuation ring $R$, whose determinant is not zero. Let $\pi$ be a uniformizing parameter in $R$. The cases that have been most studied are when $R=\mathbb{C}\{z\}$ is the ring of convergent power series in one variable, with $\pi=z$, or when $R=\mathbb{Z}_{p}$ is the ring of $p$-adic integers, with $\pi=p$. By elementary row and column operations 1 one can reduce $A$ to a diagonal matrix, with diagonal entries $\pi^{\alpha_{1}}, \pi^{\alpha_{2}}, \ldots, \pi^{\alpha_{n}}$, for unique nonnegative integers $\alpha_{1} \geq \ldots \geq \alpha_{n}$. We call $\alpha=\left(\alpha_{1}, \ldots, \alpha_{n}\right)$ the invariant factors (or Smith invariants) of $A$. The question in this case is:

Which $\alpha, \beta, \gamma$ can be the invariant factors of matrices $A, B$, and $C$ if $C=A \cdot B$ ?

In the case of convergent power series, this problem was proposed by I. Gohberg and M. A. Kaashoek, and was attacked particularly by Thompson and his coworkers (cf. [Th1, Th2], [Th3], [Thi]).

These matrices correspond to endomorphisms of $R^{n}$, with cokernels being torsion modules with at most $n$ generators. Such a module is isomorphic to a direct sum

$$
R / \pi^{\alpha_{1}} R \oplus R / \pi^{\alpha_{2}} R \oplus \ldots \oplus R / \pi^{\alpha_{n}} R, \quad \alpha_{1} \geq \ldots \geq \alpha_{n} .
$$

We call $\alpha=\left(\alpha_{1}, \ldots, \alpha_{n}\right)$ the invariant factors of the module. Denoting cokernels of $A, B$, and $C$ by $\mathcal{A}, \mathcal{B}$, and $\mathcal{C}$, one has a short exact sequence

$$
0 \rightarrow \mathcal{B} \rightarrow \mathcal{C} \rightarrow \mathcal{A} \rightarrow 0
$$

i.e., $\mathcal{B}$ is a submodule of $\mathcal{C}$, with $\mathcal{C} / \mathcal{B} \cong \mathcal{A}$. Conversely (cf. Th3]), such an exact sequence corresponds to $n$ by $n$ matrices $A, B$, and $C$ with $C=A \cdot B$. This

\footnotetext{
${ }^{1}$ The elementary row operations are: interchanging two rows, adding to any row a multiple by an element of $R$ times another row, multiplying any row by an invertible element of $R$. Similarly for elementary column operations.
} 
correspondence is seen easily by applying the "snake lemma" (see e.g. [La] §III.7) to the diagram

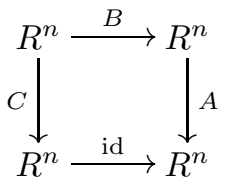

Note that the equality $(*)$, that $\sum \gamma_{i}=\sum \alpha_{i}+\sum \beta_{i}$, is obviously satisfied, since the determinant of a product is the product of the determinants. It is also not difficult to verify that inequality (1) must also be valid. Thompson [Th1 proved (6) in this setting.

When $R$ is the ring of $p$-adic integers, one is asking what finite abelian $p$-groups can appear in a short exact sequence. The aim of workers in this field was to use the numbers of submodules $\mathcal{B}$ of a given module $\mathcal{C}$ with specified invariant factors for $\mathcal{B}, \mathcal{C}$, and $\mathcal{A}=\mathcal{C} / \mathcal{B}$ as the structure constants to define a ring. In fact, this idea goes back to a lecture of E. Steinitz in 1900, but this was lost until the 1980's. From the 1940's to the 1960's, this theory was developed by P. Hall, J.A. Green, and T. Klein. The conclusion of this study that is relevant to our question was that a triple $(\alpha, \beta, \gamma)$ occurs for $p$-groups, or in fact for any discrete valuation ring, if and only if a certain nonnegative integer $c_{\alpha \beta}^{\gamma}$, called the Littlewood-Richardson coefficient, is nonzero. These coefficients, which have a purely combinatorial description, arose in representation theory and will be defined and discussed in the next section.

Although there seems to be little relation between this problem and the eigenvalue problem, they in fact have exactly the same answers. In particular, this implies the fact that the answer to this problem is independent of the discrete valuation ring.

Theorem 7. For any discrete valuation ring $R$, a triple $(\alpha, \beta, \gamma)$ occurs as the invariant factors of $A, B$, and $C=A \cdot B$ if and only if $\sum \gamma_{i}=\sum \alpha_{i}+\sum \beta_{i}$ and the inequalities $\left(*_{I J K}\right)$ are satisfied for all $(I, J, K)$ in $T_{r}^{n}$, for all $r<n$.

In fact, these results can be extended to any principal ideal domain $R$, such as the integers $\mathbb{Z}$ or a polynomial ring $F[T]$ in one variable over a field. In this case the invariant factors of a matrix $A$ with nonzero determinant are a chain $a_{1} \subset a_{2} \subset \ldots \subset a_{n}$ of nonzero ideals in $R$. The matrix $A$ can be reduced by left and right multiplication by matrices in $G L(n, R)$ to a diagonal matrix whose successive entries generate the ideals in the chain; equivalently, the cokernel of $A$ is isomorphic to $\oplus_{i=1}^{n} R / a_{i}$.

Theorem 8. For any principal ideal domain $R$, a triple $(a, b, c)$ of chains of ideals occurs as the invariant factors of $A, B$, and $C=A \cdot B$ (or of torsion modules $\mathcal{A}, \mathcal{B}$, and $\mathcal{C}$ with at most $n$ generators with $\mathcal{B} \subset \mathcal{C}$ and $\mathcal{C} / \mathcal{B} \cong \mathcal{A})$ if and only if $\prod_{i} c_{i}=\prod_{i} a_{i} \cdot \prod_{i} b_{i}$, and, for all $r<n$, and all $(I, J, K)$ in $T_{r}^{n}$, we have

$$
\prod_{k \in K} c_{k} \supset \prod_{i \in I} a_{i} \cdot \prod_{j \in J} b_{j} .
$$

One can also consider matrices with entries in the quotient field of $R$, in which case the sequences $\alpha, \beta$, and $\gamma$ may contain negative integers, and the ideals $a, b$, and $c$ may include fractional ideals. Theorems 7 and 8 extend immediately to these situations, by multiplying the matrices by scalars to get all entries in $R$. 
This theorem also gives a solution of a problem called the Carlson problem $([\mathbf{C}]$, cf. Th2, $[\mathrm{SQS} \mid$ ). The general theorem is the following:

Theorem 9. Let $A$ and $B$ be $p$ by $p$ and $q$ by $q$ matrices with entries in a principal ideal domain $R$, with nonzero determinants, and with invariant factors $a_{1} \subset \ldots \subset$ $a_{p}$ and $b_{1} \subset \ldots \subset b_{q}$. Then the possible invariant factors for a matrix of the form

$$
C=\left(\begin{array}{cc}
A & X \\
0 & B
\end{array}\right)
$$

for $X$ a $p$ by $q$ matrix with entries in $R$ are those $c_{1} \subset \ldots \subset c_{n}$, with $n=p+q$, for which $\prod_{k=1}^{n} c_{k}=\prod_{i=1}^{p} a_{i} \cdot \prod_{j=1}^{q} b_{j}$, and

$$
\prod_{k \in K} c_{k} \supset \prod_{\substack{i \in I \\ i \leq p}} a_{i} \cdot \prod_{\substack{j \in J \\ j \leq q}} b_{j}
$$

for all $(I, J, K)$ in $T_{r}^{n}$ and all $r<n$.

An arbitrary $s$ by $s$ matrix $A$ with entries in a field $F$ has invariant factors $a_{1} \subset \ldots \subset a_{p}$ in $F[T]:$ these are the invariant factors of the $F[T]$-module $F^{n}$, where $T$ acts by $T \cdot v=A v$ for $v \in F^{n}$. The following theorem solves the original Carlson problem $([\mathrm{C}])$ :

Theorem 10. Let $A$ and $B$ be $s$ by $s$ and $t$ by $t$ matrices over a field $F$. Let $a_{1} \subset \ldots \subset a_{p}$ and $b_{1} \subset \ldots \subset b_{q}$ be the invariant factors of $A$ and $B$. Then the possible invariant factors of a matrix $C=\left(\begin{array}{cc}A & X \\ 0 & B\end{array}\right)$, with $X$ an $s$ by $t$ matrix with entries in $F$, are those $c_{1} \subset \ldots \subset c_{n}$, with $n=p+q$, that satisfy the conditions of Theorem 9 .

For example, if $A=B=\left(\begin{array}{ll}0 & 1 \\ 0 & 0\end{array}\right)$, the invariant factors are $a_{1}=b_{1}=\left(T^{2}\right)$, with $p=q=1$. There are three possible invariant factors of $C:\left(T^{2}\right) \subset\left(T^{2}\right)$, which occurs with $X=\left(\begin{array}{ll}0 & 0 \\ 0 & 0\end{array}\right) ;\left(T^{3}\right) \subset(T)$, with $X=\left(\begin{array}{cc}1 & 0 \\ 0 & 0\end{array}\right)$; and $\left(T^{4}\right) \subset(1)$, with $X=\left(\begin{array}{ll}0 & 0 \\ 1 & 0\end{array}\right)$. Note that $p$ and $q$ are usually smaller than $s$ and $t$, and the matrices $A, B$, and $X$ have different meanings in Theorems 9 and 10 .

For an application, suppose $A$ is a nilpotent matrix, with Jordan blocks of sizes $\alpha_{1} \geq \alpha_{2} \geq \ldots \geq \alpha_{p}$, and $B=0$ is a $q$ by $q$ matrix. We apply Theorem 10 with $a_{i}=t^{\alpha_{i}}, b_{j}=t^{\beta_{j}}$ with $\beta_{j}=1$, and $c_{k}=t^{\gamma_{k}}$. In this case a rule of Pieri, given in equation (16) in the next section, implies that the possible $C$ have Jordan blocks of sizes $\gamma_{1} \geq \ldots \geq \gamma_{t}$, with $p \leq t \leq p+q, \sum \gamma_{i}=\sum \alpha_{i}+p, \alpha_{i}+1 \geq \gamma_{i} \geq \alpha_{i}$ for $1 \leq i \leq p$, and $\gamma_{i}=1$ for $p<i \leq t$. This result has been used by C. R. Johnson and E. A. Schreiner [JS] to give a quick proof of a theorem of H. Flanders characterizing which pairs $(C, D)$ of an $m$ by $m$ matrix $C$ and an $n$ by $n$ matrix $D$ have the form $(A \cdot B, B \cdot A)$ for some $m$ by $n$ matrix $A$ and some $n$ by $m$ matrix $\mathrm{B}$.

Unlike the situations we have seen previously, in Theorems 9 and 10 the matrices $A$ and $B$, and not merely their invariant factors, can be specified arbitrarily in advance.

The inequalities (13) also tell, for a fixed $k$ between 1 and $n$, and fixed partitions $\alpha$ and $\beta$, exactly which integers can be $\gamma_{k}$ for some triple $(\alpha, \beta, \gamma)$ that occur as invariant factors. As before, the necessity we know. To prove sufficiency it suffices to construct matrices that realize the possibilities. This has been done by J. F. Queiró and E. Marques de Sá QS. In light of the stronger results we now have, this raises the question of what can be said about other subsets. 


\section{Highest Weights}

An irreducible, finite-dimensional, holomorphic representation of $G L_{n}(\mathbb{C})$ is characterized by its highest weight, which is a weakly decreasing sequence

$$
\alpha=\left(\alpha_{1} \geq \alpha_{2} \geq \ldots \geq \alpha_{n}\right)
$$

of integers. For example, the representation $\bigwedge^{k}\left(\mathbb{C}^{n}\right)$ corresponds to the sequence $(1,1, \ldots, 1,0, \ldots, 0)$ consisting of $k 1$ 's and $n-k 0$ 's, and the representation $\operatorname{Sym}^{k}\left(\mathbb{C}^{n}\right)$ has highest weight $(k, 0, \ldots, 0)$. In general such a representation contains a nonzero vector $v$ (called a highest weight vector), such that for any upper triangular matrix $X$ in $G L_{n}(\mathbb{C})$, whose entries down the diagonal are $x_{1}, x_{2}, \ldots, x_{n}$,

$$
X \cdot v=x_{1}^{\alpha_{1}} x_{2}^{\alpha_{2}} \cdots x_{n}^{\alpha_{n}} v .
$$

We denote the irreducible representation with highest weight $\alpha$ by $V(\alpha)$. It is a basic fact of representation theory that $G L_{n}(\mathbb{C})$ is reductive. This means that any finitedimensional holomorphic representation decomposes into a direct sum of irreducible representations, and the number of times that a given irreducible representation $V(\gamma)$ appears in the sum is independent of the choice of the decomposition. In particular, for any $\alpha$ and $\beta$, the tensor product $V(\alpha) \otimes V(\beta)$ decomposes into a direct sum of representations $V(\gamma)$. Define $c_{\alpha \beta}^{\gamma}$ to be the number of copies of $V(\gamma)$ in an irreducible decomposition of $V(\alpha) \otimes V(\beta)$. The problem of interest in this situation is:

When does $V(\gamma)$ occur in $V(\alpha) \otimes V(\beta)$; i.e., when is $c_{\alpha \beta}^{\gamma}>0$ ?

It follows immediately from the definition of highest weights that a necessary condition for this is that $\sum \gamma_{i}=\sum \alpha_{i}+\sum \beta_{i}$. Other conditions are more difficult to find, although an expert may attempt to prove some of the inequalities (1) - (7).

A simple case of this problem is when $\beta=(1, \ldots, 1)$, so $V(\beta)$ is the onedimensional determinant representation. In this case $V(\alpha) \otimes V(\beta)$ is equal to $V\left(\alpha_{1}+1, \alpha_{2}+1, \ldots, \alpha_{n}+1\right)$. In particular, the problem is unchanged if each of the representations is tensored by this determinant representation several times. Therefore we may assume that each of $\alpha, \beta$, and $\gamma$ consists of nonnegative integers, i.e., is a partition. Equivalently, one need only consider polynomial representations of $G L_{n}(\mathbb{C})$. These representations were constructed in the nineteenth century by J. Deruyts [D]. For a simple construction of them, see [Fu2], §8.1.

In 1934 Littlewood and Richardson [LR] gave a remarkable combinatorial formula for the numbers $c_{\alpha \beta}^{\gamma}$, although a complete proof of the formula was only given much later (cf. [Mac, [ Fu2]). This formula is stated in terms of the Young (or Ferrers) diagrams corresponding to the partitions. The diagram of $\alpha$ is an array of boxes, lined up at the left, with $\alpha_{i}$ boxes in the $i^{\text {th }}$ row, with the rows arranged from top to bottom. For example,

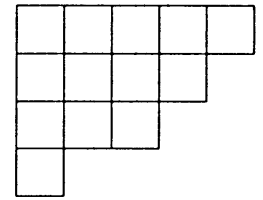

is the Young diagram of $(5,4,3,1)$. We follow the convention, reinforced by the Young diagrams, of identifying two partitions if they differ by a string of zeros at the end. 
Their rule states that $c_{\alpha \beta}^{\gamma}$ is zero unless $\sum \gamma_{i}=\sum \alpha_{i}+\sum \beta_{i}$ and the Young diagram of $\alpha$ is contained in that for $\gamma$, i.e., $\alpha_{i} \leq \gamma_{i}$ for $1 \leq i \leq n$. The complement of the Young diagram of $\alpha$ in that of $\gamma$, denoted $\gamma \backslash \alpha$, then consists of $\sum \beta_{i}$ boxes. We order the boxes of $\gamma \backslash \alpha$ by first listing the boxes in the top row, from right to left, then the boxes in the second row from right to left, and so on down the array. The Littlewood-Richardson coefficient $c_{\alpha \beta}^{\gamma}$ is the number of ways to fill the boxes of $\gamma \backslash \alpha$, with one integer in each box, so that the following four conditions are satisfied:

(i) The entries in any row are weakly increasing from left to right.

(ii) The entries in each column are strictly increasing from top to bottom.

(iii) The integer $i$ occurs exactly $\beta_{i}$ times.

(iv) For any $p$ with $1 \leq p<\sum \beta_{i}$, and any $i$ with $1 \leq i<n$, the number of times $i$ occurs in the first $p$ boxes of the ordering is at least as large as the number of times that $i+1$ occurs in these first $p$ boxes.

(The last condition says that when the entries are listed, from right to left in rows, from the top row to the bottom, they form a lattice word.)

For $\alpha=(3,2,1), \beta=(3,2,2)$, and $\gamma=(5,4,3,1)$, the following are some of the ways to fill the boxes of $\gamma \backslash \alpha$ satisfying the first three conditions.
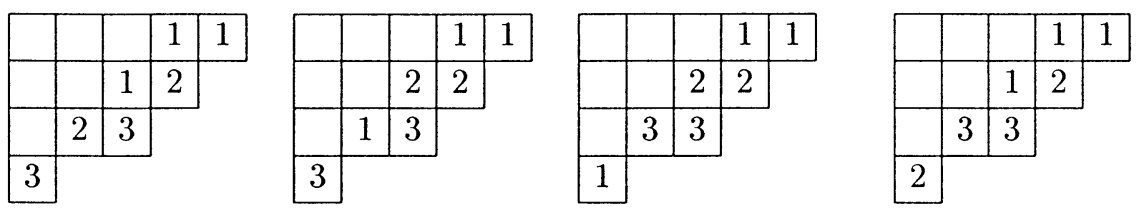

The first three examples satisfy the fourth condition. The fourth example does not, since the first six boxes in the ordering have more 3's than 2's. One sees easily that the first three are the only possibilities satisfying all four conditions, so $c_{\alpha \beta}^{\gamma}=3$.

Two special cases of this rule were known to Pieri in the context of Schubert calculus. Let $\alpha=\left(\alpha_{1}, \ldots, \alpha_{n}\right)$. If $\beta=(p)$, then the possible $\gamma$ for which $c_{\alpha \beta}^{\gamma} \neq 0$ are those of the form $\left(\gamma_{1}, \ldots, \gamma_{n+1}\right)$ with

$$
\begin{aligned}
\gamma_{1} \geq \alpha_{1} \geq \gamma_{2} \geq \alpha_{2} \geq \ldots \geq \gamma_{n} \geq & \alpha_{n} \geq \gamma_{n+1} \geq 0, \\
& \text { with } \sum \gamma_{i}=\sum \alpha_{i}+p .
\end{aligned}
$$

In these cases $c_{\alpha \beta}^{\gamma}=1$. (For representations of $G L_{n}(\mathbb{C})$ only those with $\gamma_{n+1}=0$ are allowed.) In terms of Young diagrams, $\beta$ consists of a row of $p$ boxes, and the diagram of $\gamma$ is obtained from that of $\alpha$ by adding $p$ boxes, with no two in any column.

The other Pieri rule is for $\beta=(1, \ldots, 1)$ consisting of $p 1$ 's. The possible $\gamma$ with $c_{\alpha \beta}^{\gamma} \neq 0$ also have $c_{\alpha \beta}^{\gamma}=1$, and these have the form $\gamma=\left(\gamma_{1}, \ldots, \gamma_{t}\right)$ with

$$
\alpha_{i}+1 \geq \gamma_{i} \geq \alpha_{i} \text { for all } i \text {, and } \sum \gamma_{i}=\sum \alpha_{i}+p .
$$

Here $\beta$ is a column of $p$ boxes, and the diagram of $\gamma$ is obtained from that of $\alpha$ by adding $p$ boxes, with no two in any row.

Another case that is easy to analyze directly from the Littlewood-Richardson rule is the case when $\gamma_{i}=\alpha_{i}+\beta_{i}$ for all $i$. In this case one sees that $c_{\alpha \beta}^{\gamma}=1$. These are the partitions which correspond to the triples of subsets listed in (6). 
Theorem 11. The Littlewood-Richardson coefficient $c_{\alpha \beta}^{\gamma}$ is positive exactly when $\sum \gamma_{i}=\sum \alpha_{i}+\sum \beta_{i}$ and the inequalities $\left(*_{I J K}\right)$ are valid for all $(I, J, K)$ in $T_{r}^{n}$, and all $r<n$.

Equivalently, in light of Theorem $1, c_{\alpha \beta}^{\gamma}$ is positive exactly when there is a triple of $n$ by $n$ Hermitian matrices $A, B, C=A+B$ with eigenvalues $\alpha, \beta$, and $\gamma$. For example, if there are permutations $\sigma$ and $\tau$ in $S_{n}$ such that $\alpha_{\sigma(i)}+\beta_{\tau(i)}=\gamma_{i}$ for $1 \leq i \leq n$, then $c_{\alpha \beta}^{\gamma}$ must be positive, since there are diagonal matrices $A, B$, and $C$ with these eigenvalues and $C=A+B$. For a combinatorial proof of this fact, which is a special case of the general (proven) "PRV conjecture", see [KT].

The first triple with $c_{\alpha \beta}^{\gamma}$ greater than 1, i.e., with the smallest $\sum \gamma_{i}$, has $\alpha=$ $(2,1,0), \beta=(2,1,0)$, and $\gamma=(3,2,1)$. In this case the coefficient is 2 , because there are two arrays that satisfy (i) - (iv):
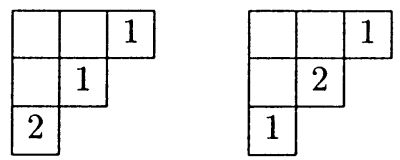

Note that this triple corresponds to the triple $(\{1,3,5\},\{1,3,5\},\{2,4,6\})$ of subsets, which appeared in examples in Section 1. The fact that this was the first triple to give a redundant Horn condition is not a coincidence. In fact, let us define $R_{r}^{n}$ to be the set of triples $(I, J, K)$ of subsets of cardinality $r$ in $\{1, \ldots, n\}$ such that the corresponding triple $(\lambda, \mu, \nu)=(\lambda(I), \lambda(J), \lambda(K))$ has $c_{\lambda \mu}^{\nu}=1$ :

$$
R_{r}^{n}=\left\{(I, J, K) \in U_{r}^{n} \mid c_{\lambda \mu}^{\nu}=1\right\} .
$$

Similarly, let

$$
S_{r}^{n}=\left\{(I, J, K) \in U_{r}^{n} \mid c_{\lambda \mu}^{\nu} \neq 0\right\} .
$$

The following theorem says that the triples occurring in Horn's conjecture are determined by the Littlewood-Richardson rule.

Theorem 12. For all $r<n$, the sets $S_{r}^{n}$ and $T_{r}^{n}$ are equal.

The sets of triples therefore have the following inclusions:

$$
R_{r}^{n} \subset S_{r}^{n}=T_{r}^{n} \subset U_{r}^{n}
$$

Theorem 13. Given that $\sum \gamma_{i}=\sum \alpha_{i}+\sum \beta_{i}$, and that each of $\alpha, \beta$, and $\gamma$ forms a weakly decreasing sequence, the conditions $\left(*_{I J K}\right)$ for all $(I, J, K)$ in $T_{r}^{n}$ and all $r<n$ are implied by the conditions $\left(*_{I J K}\right)$ for all $(I, J, K)$ in $R_{r}^{n}$ and all $r<n$.

In the theorems involving inequalities $\left(*_{I J K}\right)$, Horn's sets $T_{r}^{n}$ can therefore be replaced by the smaller sets $R_{r}^{n}$. (An indication of how much smaller these sets are can be found at the end of Section 9.) In fact, Knutson, Tao, and Woodward have announced [KTW] that the conditions $\left(*_{I J K}\right)$ for $(I, J, K)$ in $R_{r}^{n}$ are independent; i.e., none of them can be omitted.

The Littlewood-Richardson coefficients also arise from the representation theory of finite symmetric groups. The complex irreducible representations of the symmetric group $S_{a}$ are indexed by partitions of $a$; for a partition $\alpha$ of $a$, let $V_{\alpha}$ be this representation. If $\alpha$ is a partition of $a$, and $\beta$ is a partition of $b$, then the representation $V_{\alpha} \otimes V_{\beta}$ is a representation of $S_{a} \times S_{b}$. The Littlewood-Richardson number $c_{\alpha \beta}^{\gamma}$ is the number of times $V_{\gamma}$ appears in the representation of $S_{a+b}$ that 
is induced from $V_{\alpha} \otimes V_{\beta}$ by the standard inclusion of $S_{a} \times S_{b}$ in $S_{a+b}$ (cf. [Mac, $\S \mathrm{I} .7$ or [Fu2], $\S 7.3$ ). Theorems 11 and 12 therefore characterize the representations that occur in this decomposition.

If $\alpha$ and $\beta$ are given, the interval in (13) specifies exactly what can be the length $\gamma_{k}$ of the $k^{\text {th }}$ row of those $\gamma$ for which $c_{\alpha \beta}^{\gamma}$ is not zero; this follows from the equivalent problem discussed in Section 2. Again there is the interesting open question of specifying the possible lengths $\left\{\gamma_{k} \mid k \in K\right\}$ of a prescribed subset of rows of such $\gamma$.

\section{Schubert Calculus}

Let $X=\operatorname{Gr}\left(n, \mathbb{C}^{m}\right)$ be the Grassmann variety of $n$-dimensional subspaces $L$ of $\mathbb{C}^{m}$. It is a complex manifold of complex dimension $n(m-n)$. A complete flag

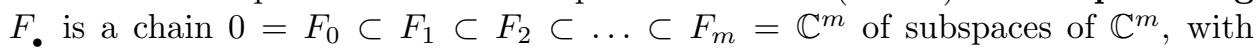
$\operatorname{dim}\left(F_{i}\right)=i$ for all $i$. For any subset $P=\left\{p_{1}<p_{2}<\ldots<p_{n}\right\}$ of cardinality $n$ in $\{1, \ldots, m\}$, there is a Schubert variety $\Omega_{P}\left(F_{\bullet}\right)$ in $X$ defined by

$$
\Omega_{P}\left(F_{\bullet}\right)=\left\{L \in X \mid \operatorname{dim}\left(L \cap F_{p_{i}}\right) \geq i \text { for } 1 \leq i \leq n\right\} .
$$

This is an irreducible closed subvariety of $X$ of dimension $\sum\left(p_{i}-i\right)$. The homology classes $\omega_{P}=\left[\Omega_{P}\left(F_{\bullet}\right)\right]$ of these varieties are independent of choice of the flag, and they form a basis for the integral homology of $X$. For each partition $\alpha$ with

$$
m-n \geq \alpha_{1} \geq \alpha_{2} \geq \ldots \geq \alpha_{n} \geq 0,
$$

let $|\alpha|=\sum \alpha_{i}$, and define $\sigma_{\alpha}$ to be the cohomology class in $H^{2|\alpha|} X$ whose cap product with the fundamental class of $X$ is the homology class $\omega_{P}$, where $P$ is defined by setting $p_{i}=m-n+i-\alpha_{i}$. Identifying cohomology and homology by this Poincaré duality isomorphism, we have:

$$
\sigma_{\alpha}=\omega_{P}, \text { where } \alpha_{i}=m-n+i-p_{i}, \quad 1 \leq i \leq n .
$$

These classes $\sigma_{\alpha}$ form a $\mathbb{Z}$-basis for the cohomology ring. It follows that for any such partitions $\alpha$ and $\beta$, there is a unique expression

$$
\sigma_{\alpha} \cdot \sigma_{\beta}=\sum d_{\alpha \beta}^{\gamma} \sigma_{\gamma}
$$

for integers $d_{\alpha \beta}^{\gamma}$, the sum over all $\gamma$ with $\sum \gamma_{i}=\sum \alpha_{i}+\sum \beta_{i}$. It is a consequence of the fact that $G L_{m}(\mathbb{C})$ acts transitively on $X$ that all these coefficients are nonnegative. The problem in this context is:

When does $\sigma_{\gamma}$ appear in the product $\sigma_{\alpha} \cdot \sigma_{\beta}$; i.e., when is the coefficient $d_{\alpha \beta}^{\gamma}$ positive?

Algebraic geometers in the nineteenth century, especially Schubert, Pieri, and Giambelli, gave algorithms for computing in this cohomology ring (in spite of the fact that cohomology was not invented until many decades later). The Giambelli formula is a determinantal expression for any $\sigma_{\alpha}$ in terms of the basic classes $\sigma_{k}$, $1 \leq k \leq m-n$, where $\sigma_{k}=\sigma_{(k)}$, and $(k)$ denotes the partition $(k, 0, \ldots, 0)$. The (original) Pieri formula (see (15)) gives the product $\sigma_{k} \cdot \sigma_{\beta}$. However, the classical geometers did not give a general closed formula for the coefficients $d_{\alpha \beta}^{\gamma}$, nor did they give a criterion for these coefficients to be positive.

Theorem 14. The class $\sigma_{\gamma}$ occurs in $\sigma_{\alpha} \cdot \sigma_{\beta}$ exactly when $\sum \gamma_{i}=\sum \alpha_{i}+\sum \beta_{i}$ and the inequalities $\left(*_{I J K}\right)$ are valid for all $(I, J, K)$ in $T_{r}^{n}$, and all $r<n$. 
This is stated for complex varieties and usual cohomology, but the same is true over any field, if one uses Chow groups instead of homology.

For later use we state another of the fundamental facts about intersection theory on Grassmannians (cf. [Fu2], §9.3). Note that

$$
H^{2 n(m-n)} X=\mathbb{Z} \cdot \sigma_{\rho},
$$

where $\rho$ consists of the integer $m-n$ repeated $n$ times; i.e., $\sigma_{\rho}=\omega_{\{1, \ldots, r\}}$ is the class of a point in $X$. We identify this top cohomology group with $\mathbb{Z}$. The dual of a class $\sigma_{\gamma}$ is the class $\sigma_{\gamma^{\prime}}$, where $\gamma^{\prime}=\left(m-n-\gamma_{n}, \ldots, m-n-\gamma_{2}, m-n-\gamma_{1}\right)$. This means that, for $|\delta|+|\gamma|=n(m-n)$, the intersection number $\sigma_{\gamma} \cdot \sigma_{\delta}$ in $H^{2 n(m-n)} X=\mathbb{Z}$ is 1 if $\delta=\gamma^{\prime}$, and 0 otherwise. Therefore, when $\sum \gamma_{i}=\sum \alpha_{i}+\sum \beta_{i}$, the class $\sigma_{\gamma}$ occurs in $\sigma_{\alpha} \cdot \sigma_{\beta}$ exactly when the product $\sigma_{\alpha} \cdot \sigma_{\beta} \cdot \sigma_{\gamma^{\prime}}$ is not zero. In fact,

$$
\sigma_{\alpha} \cdot \sigma_{\beta} \cdot \sigma_{\gamma^{\prime}}=d_{\alpha \beta}^{\gamma} \sigma_{\rho} \text {. }
$$

We will use an important fact that is part of this Schubert calculus. Suppose, for each $1 \leq s \leq k, P(s)=\left\{p_{1}(s)<\ldots<p_{n}(s)\right\}$ is a subset of $\{1, \ldots, m\}$ of cardinality $n$, and $F_{.}(s)$ is any complete flag. Then the intersection of the Schubert varieties $\bigcap_{s=1}^{k} \Omega_{P(s)}\left(F_{\bullet}(s)\right)$ must be nonempty if the corresponding product $\prod_{s=1}^{k} \omega_{P(s)}$ is not zero. This is a special case of a general fact in intersection theory, that the intersection of classes of varieties has a representative on the intersection of the varieties (cf. Fu1]). The converse is also true if the flags $F_{\bullet}(s)$ are in general position. That is, there is a dense open set (the complement of a closed algebraic subset in the product of $k$ flag varieties) of $k$-tuples of flags which are in general position. This is a special case of Kleiman's transversality theorem [Kle.

\section{Singular VAlues of Sums AND PRODUCtS}

We regard $\mathbb{C}^{m}$ as the space of column vectors, and we use the standard Hermitian inner product, denoted $(u, v)=\sum_{i=1}^{n} u_{i} \overline{v_{i}}$ for $u$ and $v$ in $\mathbb{C}^{m} ;|u|$ denotes the square root of $(u, u)$.

An arbitrary real or complex $m$ by $n$ matrix $A$ has singular values $a_{1} \geq a_{2} \geq$ $\ldots \geq a_{q} \geq 0$, where $q$ is the minimum of $m$ and $n$. They can be defined as follows. The largest singular value $a_{1}$ is the maximum of $\left|\left(A u_{1}, v_{1}\right)\right|$ as $u_{1}$ varies over unit vectors in $\mathbb{C}^{n}$, and $v_{1}$ varies over unit vectors in $\mathbb{C}^{m}$. Choosing $u_{1}$ and $v_{1}$ to achieve this maximum, define

$$
a_{2}=\operatorname{Max}_{\substack{\left|u_{2}\right|=1, u_{2} \perp u_{1} \\\left|v_{2}\right|=1, v_{2} \perp v_{1}}}\left|\left(A u_{2}, v_{2}\right)\right| .
$$

And so on for $a_{3}, \ldots, a_{q}$. In fact, there is a unitary $n$ by $n$ matrix $U$, a unitary $m$ by $m$ matrix $V$, and an $m$ by $n$ matrix $D$ that has $a_{1}, \ldots, a_{q}$ down the diagonal and is otherwise zero, such that $A=V D U^{*}$. This presentation is called a singular value decomposition of $A$. The vectors $u_{i}$ and $v_{i}$ can be taken as the $i^{\text {th }}$ columns of $U$ and $V$ respectively, for $1 \leq i \leq q$. This decomposition is important in multivariate analysis, where the entry $a_{i j}$ describes the $j^{\text {th }}$ property of an $i^{\text {th }}$ object; changing bases by $U$ and $V$ gives the best coordinates to distinguish correlations among the properties. It is also used in numerical algorithms. Geometrically, if $A$ is real, the image $A\left(S^{n-1}\right)$ of the unit sphere is an ellipsoid in $\mathbb{R}^{m}$, and the lengths of its principal axes are twice the singular values of $A$.

These singular values can also be determined in other ways. For example, if $m \leq n$, then $a_{1}^{2}, \ldots, a_{m}^{2}$ are the eigenvalues of the Hermitian matrix $A A^{*}$; if $n \leq m$, 
one can similarly use $A^{*} A$. In general, $\pm A$ and $\pm A^{*}$ have the same singular values as $A$. A characterization we will use is that the eigenvalues of the $m+n$ by $m+n$ Hermitian matrix

$$
\left(\begin{array}{cc}
0 & A \\
A^{*} & 0
\end{array}\right)
$$

are $a_{1} \geq a_{1} \geq \ldots \geq a_{q} \geq 0 \geq \ldots \geq 0 \geq-a_{q} \geq \ldots \geq-a_{2} \geq-a_{1}$, where the number of zeros in the middle is the difference between $m$ and $n$. The problem we consider in this setting is:

What $a, b$, and $c$ can be the singular values of $m$ by $n$ matrices $A$, $B$, and $C$, with $C=A+B$ ?

Given $a=\left(a_{1} \geq \ldots \geq a_{q} \geq 0\right)$, set

$$
\alpha=\left(a_{1} \geq \ldots \geq a_{q} \geq 0 \geq \ldots \geq 0 \geq-a_{q} \geq \ldots \geq-a_{1}\right),
$$

a nonincreasing sequence of length $m+n$, and define similarly sequences $\beta$ and $\gamma$ from $b$ and $c$. For each triple $(I, J, K)$ of subsets of $\{1, \ldots, m+n\}$ of the same cardinality $r<m+n$, the inequality $\left(*_{I J K}\right)$ on $\alpha, \beta$, and $\gamma$ determines a corresponding inequality on $a, b$, and $c$, that we denote by $\left(*_{I J K}\right)$. For example, if $m=n=2, r=1$, and $I=\{3\}, J=\{1\}, K=\{3\}$, the inequality $\gamma_{3} \leq \alpha_{3}+\beta_{1}$ becomes the inequality $-c_{2} \leq-a_{2}+b_{1}$, or $a_{2} \leq c_{2}+b_{1}$. In general, setting $I^{\prime}=\{i \in\{1, \ldots, m+n\} \mid m+n+1-i \in I\}$, and similarly for $J$ and $K$, the inequality is

$\left(* *_{I J K}\right)$

$$
\sum_{\substack{k \in K \\ k \leq q}} c_{k}-\sum_{\substack{k \in K^{\prime} \\ k \leq q}} c_{k} \leq \sum_{\substack{i \in I \\ i \leq q}} a_{i}-\sum_{\substack{i \in I^{\prime} \\ i \leq q}} a_{i}+\sum_{\substack{j \in J \\ j \leq q}} b_{j}-\sum_{\substack{j \in J^{\prime} \\ j \leq q}} b_{j} .
$$

Theorem 15. A triple $a, b, c$ occurs as singular values of $m$ by $n$ complex matrices $A, B$, and $C$ with $C=A+B$, if and only if the inequalities (**IJK) are satisfied for all $(I, J, K)$ in $T_{r}^{m+n}$, for all $r<m+n$.

Many special cases of the necessity of these conditions had been known, cf. [AM], [TT1], QS].

In contrast to the eigenvalue problem discussed in the first paragraph, the situation for real matrices is not the same as that for complex matrices. For 1 by 1 complex matrices, a triple of nonnegative numbers occurs whenever each is at most the sum of the other two; for real matrices, one must be equal to the sum of the other two. Here is a more interesting example:

Example 4. For 3 by 3 matrices, the triple $a=(1,1,0), b=(1,1,0)$, and $c=$ $(1,1,1)$ occurs as singular values of complex matrices $A, B$ and $C=A+B$, but they do not occur for real matrices. In the complex case, $A$ and $B$ can be diagonal matrices with entries $(1, \zeta, 0)$ and $\left(0, \zeta^{-1}, 1\right)$, with $\zeta$ a primitive $6^{\text {th }}$ root of unity. It is a straightforward calculation to verify that they do not occur for real matrices.

When one writes out the inequalities of Theorem 15, one finds that most of them are redundant and that the essential inequalities remaining have quite a simple form. For example, for 4 by 4 matrices, the triple $(\{1,3,5\},\{2,3,5\},\{2,4,7\})$ in $T_{3}^{8}$ gives the inequality $c_{2}+c_{4}-c_{2} \leq a_{1}+a_{3}-a_{4}+b_{2}+b_{3}-b_{4}$. This inequality follows from the inequality $c_{4} \leq a_{3}+b_{2}$ and the inequalities $a_{4} \leq a_{1}$ and $b_{4} \leq b_{3}$. The triple $(\{3,7\},\{2,3\},\{4,8\})$ in $T_{2}^{8}$, on the other hand, gives the inequality $c_{4}-c_{1} \leq$ 
$a_{3}-a_{2}+b_{2}+b_{3}$, or

$$
a_{2}+c_{4} \leq c_{1}+a_{3}+b_{2}+b_{3}
$$

which is in fact essential: it determines one of the facets of the cone defined in Theorem 15.

For simplicity, let us consider only $n$ by $n$ matrices. For $n=1$, minimal inequalities defining the possible singular values are: $c_{1} \leq a_{1}+b_{1}, b_{1} \leq a_{1}+c_{1}$, and $a_{1} \leq b_{1}+c_{1}$. (In this case the inequalities $a_{1} \geq 0, b_{1} \geq 0$, and $c_{1} \geq 0$ follow.) For $n \geq 2$, Buch has a precise conjecture for producing a minimal set of inequalities: they are the $3 n$ inequalities

$(* *) \quad a_{1} \geq a_{2} \geq \ldots \geq a_{n} \geq 0, b_{1} \geq b_{2} \geq \ldots \geq b_{n} \geq 0, c_{1} \geq c_{2} \geq \ldots \geq c_{n} \geq 0$,

and the inequalities $\left(* *_{I J K}\right)$ coming from the following set of triples: The triple $(I, J, K)$ must be in $R_{r}^{2 n}$, and the following two conditions must be satisfied:

(i) None of $I, J$, or $K$ contains a pair of the form $\{t, 2 n+1-t\}$ for any $1 \leq t \leq n$.

This means that there is no cancellation when one writes out the corresponding inequality $\left(* *_{I J K}\right)$. In particular, this requires $r$ to be no larger than $n$. For a subset $I$ of $\{1, \ldots, 2 n\}$ of cardinality $r$, not containing any pair $\{t, 2 n+1-t\}$, set

$$
\bar{I}=\{i \in\{1, \ldots, n\} \mid i \in I \text { or } 2 n+1-i \in I\}=\left\{\bar{i}_{1}<\bar{i}_{2}<\ldots<\bar{i}_{r}\right\},
$$

and define $\bar{J}$ and $\bar{K}$ similarly from $J$ and $K$. The second condition is

(ii) $\left\{p \leq r \mid \bar{k}_{p} \notin K\right\}=\left\{p \leq r \mid \bar{i}_{p} \notin I\right\} \amalg\left\{p \leq r \mid \bar{j}_{p} \notin J\right\}$,

where $\amalg$ denotes disjoint union. This condition guarantees that when the inequality $\left(* *_{I J K}\right)$ is written out as an inequality with all positive coefficients, as we did in the example (24), there are $r$ terms on the left and $2 r$ terms on the right, and, for each position $p$ between 1 and $r$, exactly one of the terms $\left\{a_{\bar{i}_{p}}, b_{\bar{j}_{p}}, c_{\bar{k}_{p}}\right\}$ is on the left, and the other two are on the right.

Conjecture (Buch). For $n \geq 2$, the inequalities $(* *)$ and $\left(* *_{I J K}\right)$, for $(I, J, K)$ in $R_{r}^{2 n}, r \leq n$, satisfying (i) and (ii), define the facets of the cone of singular values for $n$ by $n$ matrices.

Buch has verified this conjecture for $n \leq 4$. Independently, L. O'Shea and R. Sjamaar [OS] have speculated how a complete set of inequalities can be obtained by looking at Schubert calculus with $\mathbb{Z} / 2 \mathbb{Z}$ coefficients in real flag varieties.

In the case of square $n$ by $n$ matrices $A, B$, and $C$, one may also ask the similar question for products:

What $a, b$, and $c$ can be the singular values of $A, B$ and $C$ with $C=A \cdot B$ ?

The answer again is controlled by the triples in $T_{r}^{n}\left(\right.$ or $\left.R_{r}^{n}\right)$ :

Theorem 16. A triple $(a, b, c)$ occurs as singular values of $n$ by $n$ matrices $A, B$, and $C=A \cdot B$ if and only if

$$
\prod_{k \in K} c_{k} \leq \prod_{i \in I} a_{i} \cdot \prod_{j \in J} b_{j}
$$

for all $(I, J, K)$ in $T_{r}^{n}$, and all $r<n$. 
In 1950 Gel'fand and Naimark GN] proved the special case of this theorem when $K=I$ and $J=\{1, \ldots, r\}$. Many other special cases have been found since then, cf. TT1].

As in the Hermitian case (Theorem 6), these results also extend to singular values of compact operators on a Hilbert space.

For the question of which $c_{k}$, for fixed $k$, can be $k^{\text {th }}$ singular values of sums or products of matrices with given singular values, the answers are similar to the Hermitian case discussed in Section 1. For these results, see QS.

\section{First STEPS TOWARD THE PROOFS}

We now start on the proofs of the theorems, together with some generalizations and complements. In contrast to the first five sections, from now on we will prove the propositions as we go along and include references to the original articles.

6.1. The Rayleigh trace. An important key to the understanding of the eigenvalue problem was given in 1962 by J. Hersch and B. P. Zwahlen, based on then recent work of A. R. Amir-Moéz and Wielandt, with ideas going back to Poincaré, Weyl, Courant, and Fischer. If $A$ is a Hermitian $n$ by $n$ matrix, with eigenvalues $\alpha$, choose corresponding orthogonal eigenvectors $v_{i}$ so $A v_{i}=\alpha_{i} v_{i}$. Let $F_{k}(A)$ be the span of $v_{1}, \ldots, v_{k}$, so we have a complete flag $F_{\text {. }}(A)$ of $\mathbb{C}^{n}$ (determined uniquely by $A$ only if its eigenvalues are distinct). For any linear subspace $L$ of $\mathbb{C}^{n}$, define the Rayleigh 2 trace $R_{A}(L)$ by

$$
R_{A}(L)=\sum_{i=1}^{r}\left(A u_{i}, u_{i}\right)
$$

where $u_{1}, \ldots, u_{r}$ is an orthonormal basis of $L$. This is independent of the choice of basis; indeed, $R_{A}(L)$ is the trace of the composite $L \rightarrow \mathbb{C}^{n} \rightarrow \mathbb{C}^{n} \rightarrow L$, where the first map is the inclusion, the second is given by $A$, and the third is orthogonal projection. The key fact, which links the sums occurring in the inequalities for the eigenvalues to Schubert varieties, is:

Proposition 1 ([HZ]). For any subset $I=\left\{i_{1}<\ldots<i_{r}\right\}$ of $\{1, \ldots, n\}$ of cardinality $r$,

$$
\sum_{i \in I} \alpha_{i}=\operatorname{Min}_{L \in \Omega_{I}\left(F_{\bullet}(A)\right)} R_{A}(L) .
$$

The proof is straightforward. For any $L$ in the Schubert variety $\Omega_{I}\left(F_{\bullet}(A)\right)$, and for any unit vector $u_{1}$ in $L \cap F_{i_{1}}(A)$, we have $\left(A u_{1}, u_{1}\right) \geq \alpha_{i_{1}}$. For any unit vector $u_{2}$ perpendicular to $u_{1}$ in $L \cap F_{i_{2}}(A)$, we have $\left(A u_{2}, u_{2}\right) \geq \alpha_{i_{2}}$. Continuing in this way, one sees that $R_{A}(L)=\sum_{p=1}^{r}\left(A u_{p}, u_{p}\right) \geq \sum_{i \in I} \alpha_{i}$. This inequality will be strict unless these choices can be made with $A u_{p}=\alpha_{i_{p}} u_{p}$ for $1 \leq p \leq r$. In particular, taking $L$ spanned by $u_{p}=v_{i_{p}}$ for $1 \leq p \leq r$, one obtains equality.

For later use we note the following consequence of this argument.

Corollary 1. For $L$ in $\Omega_{I}\left(F_{\bullet}(A)\right)$, the only way the equality $\sum_{i \in I} \alpha_{i}=R_{A}(L)$ can hold is if $L$ is spanned by eigenvectors $u_{1}, \ldots, u_{r}$ such that $A\left(u_{p}\right)=\alpha_{i_{p}} u_{p}$ for $1 \leq p \leq r$. In this case $A$ maps $L$ (and therefore also $L^{\perp}$ ) to itself. If the

\footnotetext{
${ }^{2}$ Lord J. W. Rayleigh, a British physicist, used the ratios $(A v, v) /(v, v)$ in physical problems involving eigenvalues. For example, the maximum (resp. minimum) of this ratio for all nonzero $v$ is the biggest (resp. smallest) eigenvalue of $A$.
} 
eigenvalues $\alpha_{1}, \ldots, \alpha_{n}$ are distinct, then there is only one $L$ in $\Omega_{I}\left(F_{\bullet}(A)\right)$ for which $\sum_{i \in I} \alpha_{i}=R_{A}(L)$.

The following two corollaries are proved by the same methods, although they are not really needed here.

Corollary 2. Let $F_{\bullet}^{\prime}(A)$ be the flag with $F_{k}^{\prime}(A)$ spanned by the last $k$ eigenvectors $v_{n+1-k}, \ldots, v_{n}$, and set $I^{\prime}=\{i \mid n+1-i \in I\}$. Then

$$
\sum_{i \in I} \alpha_{i}=\operatorname{Max}_{L \in \Omega_{I^{\prime}}\left(F_{\bullet}^{\prime}(A)\right)} R_{A}(L) .
$$

To prove this, construct a sequence of orthonormal vectors $u_{p}$ in $L \cap F_{i_{p}^{\prime}}^{\prime}(A)$. One has $\left(A u_{p}, u_{p}\right) \leq \alpha_{i_{r+1-p}}$ for $1 \leq p \leq r$. Taking $L$ spanned by $v_{i_{1}}, \ldots, v_{i_{r}}$ gives equality.

Corollary 3. Let $A_{L}: L \rightarrow L$ denote the composite $L \rightarrow \mathbb{C}^{n} \rightarrow \mathbb{C}^{n} \rightarrow L$ defined before the proposition, and let $\alpha_{1}^{L} \geq \ldots \geq \alpha_{r}^{L}$ be its eigenvectors. Then

$$
\alpha_{n-r+k} \leq \alpha_{k}^{L} \leq \alpha_{k} \quad \text { for } \quad 1 \leq k \leq r .
$$

For this, let $w_{1}, \ldots, w_{r}$ be an orthonormal basis of $L$ with $A_{L}\left(w_{i}\right)=\alpha_{i}^{L} w_{i}$, $1 \leq i \leq r$. The intersection

$$
\left\langle w_{1}, \ldots, w_{k}\right\rangle \cap\left\langle v_{1}, \ldots, v_{k-1}\right\rangle^{\perp}=\left\langle w_{1}, \ldots, w_{k}\right\rangle \cap\left\langle v_{k}, \ldots, v_{n}\right\rangle
$$

is not empty, and if $v$ is a unit vector in it, then $\alpha_{k}^{L} \leq(A v, v) \leq \alpha_{k}$. Taking a unit vector in $\left\langle w_{k}, \ldots, w_{r}\right\rangle \cap\left\langle v_{1}, \ldots, v_{n-r+k}\right\rangle$ gives the other inequality.

When $L$ is the subspace spanned by some of the basic vectors of $\mathbb{C}^{n}$, the matrix for $A_{L}$ is a principal minor of $A$, and the result of Corollary 3 is sometimes called the inclusion principle.

With a little Schubert calculus, Proposition 1 leads quickly to a proof of the following proposition.

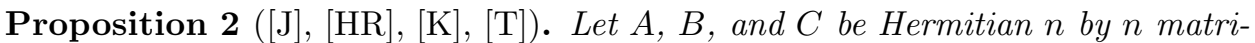
ces, with $C=A+B$, and eigenvalues $\alpha, \beta$, and $\gamma$. Then for any $r<n$ and any $(I, J, K)$ in $S_{r}^{n}$, the inequality $\left(*_{I J K}\right)$ is valid.

To prove this, note first that the Rayleigh trace is linear in the matrices, so

$$
R_{-A}(L)+R_{-B}(L)+R_{C}(L)=R_{0}(L)=0 .
$$

Note also that if $\alpha_{1} \geq \ldots \geq \alpha_{n}$ are the eigenvalues of $A$, then $\alpha_{1}^{\prime}=-\alpha_{n} \geq$ $\ldots \geq \alpha_{n}^{\prime}=-\alpha_{1}$ are the eigenvalues of $-A$. For a subset $I$ of $\{1, \ldots, n\}$, let $I^{\prime}=\{i \mid n+1-i \in I\}$. This gives the equations

$$
\sum_{i \in I^{\prime}} \alpha_{i}^{\prime}=-\sum_{i \in I} \alpha_{i} .
$$

It follows from the definition of Schubert classes (see (21)) that

$$
\sigma_{\lambda(I)}=\omega_{I^{\prime}}
$$

where the notation is that of Section 4, but working now in the Grassmannian $G r\left(r, \mathbb{C}^{n}\right)$. In addition,

$$
\sigma_{\lambda(I)} \text { and } \sigma_{\lambda\left(I^{\prime}\right)} \text { are dual. }
$$

This means that $\sigma_{\lambda(I)} \cdot \sigma_{\lambda\left(J^{\prime}\right)}=\sigma_{\rho}$ if and only if $I=J$, where $\rho=(n-r)^{r}$, so $\sigma_{\rho}$ is the class of a point. The following are therefore equivalent: 
(i) $\sigma_{\lambda(K)}$ occurs in $\sigma_{\lambda(I)} \cdot \sigma_{\lambda(J)}$, i.e., $(I, J, K) \in S_{r}^{n}$;

(ii) $\sigma_{\lambda(I)} \cdot \sigma_{\lambda(J)} \cdot \sigma_{\lambda\left(K^{\prime}\right)} \neq 0$;

(iii) $\omega_{I^{\prime}} \cdot \omega_{J^{\prime}} \cdot \omega_{K} \neq 0$.

This last condition implies that the intersection $\Omega_{I^{\prime}}\left(F_{\bullet}(-A)\right) \cap \Omega_{J^{\prime}}\left(F_{\bullet}(-B)\right) \cap$ $\Omega_{K}(F$. $(C))$ is not empty. Taking $L$ in this intersection gives

$$
-\sum_{i \in I} \alpha_{i}-\sum_{j \in J} \beta_{j}+\sum_{k \in K} \gamma_{k} \leq R_{-A}(L)+R_{-B}(L)+R_{C}(L)=0,
$$

and this proves Proposition 2.

Remark. Using the equation $A+B+(-C)=0$, one deduces by a similar argument that $\sum_{k \in K^{\prime}} \gamma_{k} \leq \sum_{i \in I^{\prime}} \alpha_{i}+\sum_{j \in J^{\prime}} \beta_{j}$, where $I^{\prime}=\{i \mid n+1-i \notin I\}$. In fact, however, a triple $(I, J, K)$ is in $S_{r}^{n}$ if and only if $\left(I^{\prime}, J^{\prime}, K^{\prime}\right)$ is in $S_{n-r}^{n}$. This follows from the isomorphism between $\operatorname{Gr}\left(r, \mathbb{C}^{n}\right)$ and $G r\left(n-r, \mathbb{C}^{n}\right)$ that takes a subspace $L$ of $\mathbb{C}^{n}$ to the kernel of the map $\mathbb{C}^{n}=\left(\mathbb{C}^{n}\right)^{*} \rightarrow L^{*}$. On cohomology this map takes $\sigma_{\lambda}$ to $\sigma_{\lambda^{\prime}}$, where $\lambda^{\prime}$ is the conjugate of $\lambda$. Note that $\lambda\left(I^{\prime}\right)$ is the transpose of $\lambda$ (see Lemma 4 in Section 10).

In 1962, and for some time after that, people working on this problem were not familiar with Schubert calculus, and it was only in 1979, in an unpublished thesis of S. Johnson [J], directed by Thompson, that the connection with Schubert calculus was made. Proposition 2 was later rediscovered by U. Helmke and J. Rosenthal [HR], Totaro [T] (in a different context), and Klyachko Kl1]. Many papers proved special cases of the inequalities $\left(*_{I J K}\right)$ by showing by hand - using only linear algebra - that the intersection of Schubert varieties appearing in the above proof must be nonempty.

6.2. Relations among the four main problems. The fact that the representation theory problem is equivalent to the Schubert calculus problem has been known at least since 1947, when L. Lesieur [Le proved that the Littlewood-Richardson coefficients $c_{\alpha \beta}^{\gamma}$ are the same as the coefficients $d_{\alpha \beta}^{\gamma}$ that describe the multiplication in the cohomology of the Grassmannian. In spite of all the known relations between Schubert varieties and representation theory, however, the proof is not very direct. It proceeds by showing that both are controlled by the same algebra of symmetric functions. The character of the representation $V(\alpha)$ of $G L_{n}(\mathbb{C})$ is the Schur polynomial $s_{\alpha}\left(x_{1}, \ldots, x_{n}\right)$. Corresponding to the decomposition of a tensor product one has the identity $s_{\alpha}(x) \cdot s_{\beta}(x)=\sum c_{\alpha \beta}^{\gamma} s_{\gamma}(x)$. These Schur polynomials form a basis for the ring $\Lambda_{n}$ of symmetric polynomials in the variables $x_{1}, \ldots, x_{n}$ (cf. [Mac], Fu2]). On the other hand, there is a surjection of the ring $\Lambda_{n}$ onto the cohomology of the Grassmannian $\operatorname{Gr}\left(n, \mathbb{C}^{m}\right)$ that takes $s_{\alpha}(x)$ to the class $\sigma_{\alpha}$. The classical formulas of Pieri and Giambelli imply that this is a ring homomorphism, which proves the following proposition (see e.g. [Fu2] for this story):

Proposition 3. The coefficients for multiplying Schubert classes are the same as those for decomposing tensor products, i.e., $d_{\alpha \beta}^{\gamma}=c_{\alpha \beta}^{\gamma}$.

Closer relations are known between the cohomology of the Grassmann variety $G / P$ and representations of $G$ stemming from work of Ehresmann and Kostant (see [Ko], $\S 8)$, but one would like a more direct explanation for the equality of Proposition 3 .

Based on the work of Hall and Green, in 1968 Klein [K] completed the proof of the following proposition: 
Proposition 4. If $R$ is a discrete valuation ring, and $\mathcal{C}$ is an $R$-module with invariant factors $\gamma$, then there is a submodule $\mathcal{B}$ of $\mathcal{C}$ with invariant factors $\beta$ such that $\mathcal{A}=\mathcal{C} / \mathcal{B}$ has invariant factors $\alpha$ if and only if the Littlewood-Richardson coefficient $c_{\alpha \beta}^{\gamma}$ is positive.

Although this establishes a relation between invariant factors and representation theory, the proof does nothing of the sort. Rather, it provides a link between such submodules and the Littlewood-Richardson algorithm. In fact, if $\mathcal{B}$ is a submodule of $\mathcal{C}$, the invariant factors $\gamma(k)$ of $\mathcal{C} / \pi^{k} \mathcal{B}$ form a chain of partitions $\alpha=\gamma(0) \subset$ $\gamma(1) \subset \ldots \subset \gamma(t)=\gamma$. If a $k$ is placed in each box of $\gamma(k) \backslash \gamma(k-1)$, Green showed that one obtains an array whose conjugate - obtained by interchanging rows and columns - satisfies the conditions (i) - (iv) of Section 2 for the conjugate partitions $\alpha^{\prime}, \beta^{\prime}, \gamma^{\prime}$. Conversely, Klein showed that any such chain of partitions can be realized by some submodule $\mathcal{B}$ of $\mathcal{C}$. To complete the proof, one needs the fact that $c_{\alpha^{\prime} \beta^{\prime}}^{\gamma^{\prime}}=c_{\alpha \beta}^{\gamma}$ (see Lemma 4 in Section 10).

There are polynomials $g_{\alpha \beta}^{\gamma}(T)$, called Hall polynomials, whose top coefficient is $c_{\alpha \beta}^{\gamma}$, such that whenever the residue field of $R$ is finite of cardinality $q$, then $g_{\alpha \beta}^{\gamma}(q)$ is the number of submodules $\mathcal{B}$ of $\mathcal{C}$ with invariant factors $\beta$, such that $\mathcal{C} / \mathcal{B}$ has invariant factors $\alpha$. For a proof, with references, applications, and history, see Chapter II of $\mathrm{Mac}$.

Thompson and his coworkers, who worked on both problems, noticed that the answers to the Hermitian eigenvalue problem and the invariant factor problem appeared to be similar to each other and were therefore related to LittlewoodRichardson coefficients by means of Proposition 4.

Although this was only realized in 1997 by A. P. Santana, J. F. Queiró, and E. Marques de Sá SQS , the following relation can be deduced from a 1982 theorem of G. J. Heckman [He] about moment mappings and coadjoint orbits:

Proposition 5. If $\alpha, \beta$, and $\gamma$ are partitions of lengths at most $n$ such that $c_{\alpha \beta}^{\gamma} \neq$ 0 , then there are Hermitian $n$ by $n$ matrices $A, B$, and $C$ with eigenvalues $\alpha, \beta$, and $\gamma$ and $C=A+B$.

In fact, we will not need this proposition; it will follow from the other results proved here. We will discuss the coadjoint orbit and moment map approach in Section 8. Note, however, that with these five propositions, relations are established among all four of our subjects. For partitions $\alpha, \beta$, and $\gamma$ of lengths at most $n$, consider the following conditions:

(i) $(\alpha, \beta, \gamma)$ occur as eigenvalues of $n$ by $n$ Hermitian matrices $A, B$, and $C=$ $A+B$

(ii) $(\alpha, \beta, \gamma)$ occur as invariant factors (over one or every discrete valuation ring) of $n$ by $n$ matrices $A, B$, and $C=A \cdot B$;

(iii) the representation $V(\gamma)$ of $G L_{n}(\mathbb{C})$ occurs in $V(\alpha) \otimes V(\beta)$;

(iv) $\sigma_{\gamma}$ occurs in $\sigma_{\alpha} \cdot \sigma_{\beta}$ in the cohomology of $G r\left(n, \mathbb{C}^{m}\right)$ for any large $m$.

Propositions 3 and 4 say that (ii), (iii), and (iv) are equivalent, and Proposition 5 says that they imply (i). See $\mathrm{SQS}]$ for more about these relations. 
Note that the Littlewood-Richardson coefficients measure a multiplicity in each of the problems (ii), (iii), and (iv). It would be interesting to find a similar interpretation for the eigenvalue problem (i). 3

Proposition 2 established a link in one direction between the eigenvalue problem for $n$ by $n$ matrices and Schubert calculus in smaller Grassmann varieties $\operatorname{Gr}\left(r, \mathbb{C}^{n}\right)$. The next main step is to find a converse to this proposition. It should be pointed out at this point that it is not obvious that any of the four problems have solutions that can be described by any inequalities of the form $\left(*_{I J K}\right)$. The fact that the eigenvalue problem can be described by some linear inequalities also follows from convexity properties of moment maps, which we will discuss in Section 8.

\section{Filtered Vector spaces, GEOMETRIC INVARIANT THEORY, AND STABILITY}

We start by describing a theorem of Totaro [T], which, although written for quite another purpose, quickly yields the converse to Proposition 2. I thank R. Lazarsfeld for pointing me to this work of Totaro. Let $V$ be an $n$-dimensional complex vector space. By a filtration $V$ of $V$ we shall mean a weakly decreasing sequence of subspaces

$$
V=V^{0} \supset V^{1} \supset V^{2} \supset \ldots \supset V^{p} \supset V^{p+1} \supset \ldots
$$

with the assumption that $V^{q}=0$ for sufficiently large $q$. Any subspace $L$ of $V$ gets an induced filtration, by setting $L^{p}=L \cap V^{p}$.

By an $m$-filtration of $V$ is meant a collection $V \cdot(s)$ of filtrations of $V$, for $1 \leq s \leq m$. For any nonzero subspace $L$ of $V$, define its slope $\mu(L)$ with respect to this $m$-filtration by

$$
\mu(L)=\frac{1}{\operatorname{dim}(L)} \sum_{s=1}^{m} \sum_{p \geq 1} \operatorname{dim}\left(L \cap V^{p}(s)\right) .
$$

Call the $m$-filtration semistable (resp. stable) if $\mu(L) \leq \mu(V)$ (resp. $\mu(L)<$ $\mu(V))$ for all subspaces $L$ of $V, 0 \neq L \neq V$. There is an obvious notion of a direct sum of $m$-filtered vector spaces, taking the $p^{\text {th }}$ subspace of the sum to be the sum of the $p^{\text {th }}$ subspaces of the factors. An $m$-filtration is called polystable if it is a direct sum of a finite number of stable $m$-filtrations of the same slope.

Proposition 6 ([T] $)$. An $m$-filtration on $V$ is polystable if and only if there is a Hermitian metric on $V$ such that the sum of the orthogonal projections from $V$ onto the spaces $V^{p}(s)$, for $p \geq 1$ and $1 \leq s \leq m$, is the scalar operator $\mu(V)$.

The proof of this uses some geometric invariant theory. Totaro shows that a polystable $m$-filtration corresponds to a polystable point $x$ in a product $X$ of $m$ partial flag varieties, which has a canonical embedding in a projective space $\mathbb{P}(E)$, where $E=\otimes_{s=1}^{m} \otimes_{p \geq 1} \bigwedge^{\operatorname{dim} V^{p}(s)} V$. It is a result of G. Kempf and L. Ness from Geometric Invariant Theory, that if $\widetilde{x}$ is a representative of $x$ in $E$, polystability is equivalent to the corresponding orbit $S L(V) \cdot \widetilde{x}$ being closed in $E$. One then chooses a Hermitian metric on $V$ (which induces a metric on $E$ ) to minimize the

\footnotetext{
${ }^{3}$ Knutson reports that there is an interpretation of a suitable volume of a space of Hermitian matrices $(A, B, C)$ with $C=A+B$ and eigenvalues $\alpha, \beta$, and $\gamma$, in terms of Littlewood-Richardson coefficients, but asymptotically, using triples $(N \alpha, N \beta, N \gamma)$ for large $N$. See also [DRW], §3.
} 
distance from $\widetilde{x}$ to the origin. The fact that this is a critical point implies, by a calculation, the asserted fact that the sum of the projections is a scalar.

This proposition was used by Totaro to give a simpler proof of a theorem of G. Faltings, that the tensor product of semistable filtrations is semistable. Essentially the same argument was found by Klyachko [K11] for the eigenvalue problem. See Section 8 and [Fu3] for more details.

If $\alpha=\left(\alpha_{1} \geq \alpha_{2} \geq \ldots \geq \alpha_{n} \geq 0\right)$ is a partition of length at most $n$, and if $F_{\text {. }}: 0=F_{0} \subset F_{1} \subset \ldots \subset F_{n}=V$ is a complete flag in $V$, define a filtration $V \bullet$ of $V$ by setting $V^{p}=F_{\alpha^{\prime}}$, where $\alpha^{\prime}$ is the conjugate partition to $\alpha$; i.e., $\alpha^{\prime}{ }_{p}$ is the cardinality of the set $\left\{i \in\{1, \ldots, n\} \mid \alpha_{i} \geq p\right\}$. Any filtration arises in this way, for some unique $\alpha$ and some flag $F_{\bullet}$, although the flag is not unique unless $\alpha$ consists of $n$ distinct integers. The following lemma is immediate from this definition.

Lemma 1. Choose a Hermitian metric on $V$, and let $A$ be the sum of the orthogonal projections of $V$ on $V^{p}$, for $p \geq 1$. Then $A$ is a Hermitian operator whose eigenvalues are $\alpha_{1}, \ldots, \alpha_{n}$.

For any subset $I=\left\{i_{1}<\ldots<i_{r}\right\}$ of $\{1, \ldots, n\}$ of cardinality $r$, we have the Schubert cell $\Omega_{I}^{\circ}\left(F_{\text {. }}\right)$, defined by

$$
\Omega_{I}^{\circ}\left(F_{\bullet}\right)=\left\{L \in G r(r, V) \mid \operatorname{dim}\left(L \cap F_{j}\right)=k \text { for } i_{k} \leq j<i_{k+1}, 0 \leq k \leq r\right\},
$$

where $i_{0}$ is defined to be 0 and $i_{r+1}$ is defined to be $n+1$; in other words, $I$ lists those $i$ for which $L \cap F_{i} \neq L \cap F_{i-1}$. This cell is a dense open subset of the Schubert variety $\Omega_{I}\left(F_{\bullet}\right)$. Any subspace belongs to a unique Schubert cell, when the flag $F$. is fixed. From this definition it follows that, for any $\alpha_{1}, \ldots, \alpha_{n}$,

$$
\sum_{i \in I} \alpha_{i}=\sum_{i=1}^{n} \alpha_{i} \operatorname{dim}\left(L \cap F_{i} / L \cap F_{i-1}\right)=\sum_{i=1}^{n}\left(\alpha_{i}-\alpha_{i+1}\right) \operatorname{dim}\left(L \cap F_{i}\right),
$$

where $\alpha_{n+1}$ is defined to be 0 . By a simple counting argument, this implies:

Lemma 2. Let $L$ be a subspace of $V$ that is in the Schubert cell $\Omega_{I}^{\circ}\left(F_{\bullet}\right)$. Then $\sum_{i \in I} \alpha_{i}=\sum_{p \geq 1} \operatorname{dim}\left(L \cap V^{p}\right)$.

We define $S_{r}^{n}(m)$ to be the set of $m$-tuples $\mathcal{I}=(I(1), \ldots, I(m))$ such that $\prod_{s=1}^{m} \omega_{I(s)} \neq 0$. Here, as in Section $4, \omega_{I}$ denotes the class of a Schubert variety $\Omega_{I}\left(F_{\bullet}\right)$.

Consider now $m$-tuples $\alpha(1), \ldots, \alpha(m)$, with each $\alpha(s)$ a nonincreasing sequence of real numbers of length at most $n$, written $\alpha(s)=\left(\alpha_{1}(s), \ldots, \alpha_{n}(s)\right)$. For any $m$-tuple $\mathcal{I}=(I(1), \ldots, I(m))$ of subsets of $\{1, \ldots, n\}$ of cardinality $r$ we have the corresponding inequality

$$
\frac{1}{r} \sum_{s=1}^{m} \sum_{i \in I(s)} \alpha_{i}(s) \leq \frac{1}{n} \sum_{s=1}^{m} \sum_{i=1}^{n} \alpha_{i}(s) .
$$

Lemma 3. Let $\alpha(s)$ be a partition of length at most $n$, for $1 \leq s \leq m$. Let $F$. $(s)$ be general flags in $V, 1 \leq s \leq m$, and let $V \cdot(s)$ be the filtration defined by the flag $F .(s)$ and the partition $\alpha(s)$, for $1 \leq s \leq m$. This $m$-filtration is semistable (resp. stable) if and only if the inequality $\left(*_{\mathcal{I}}\right)$ holds (resp. with strict inequality) for every $\mathcal{I}$ in $S_{r}^{n}(m)$ and all $r \leq n$.

This follows from Lemma 2 and the fact that, when the $F_{\text {. }}(s)$ are general flags, an intersection of Schubert cells $\bigcap_{s=1}^{m} \Omega_{I(s)}^{\circ}\left(F_{\bullet}(s)\right)$ is nonempty if and only if the corresponding class $\prod_{s=1}^{m} \omega_{I(s)}$ is not zero. 
The following, in the Hermitian case, is one of the main results of Klyachko's paper Kl1. From now on, we will state the results for an arbitrary number of factors, rather than the three that were featured in the first section, and we will put them all on the same side of the equation. This allows a simpler and more natural expression of the results and proofs, by avoiding the kind of manipulations that we saw in the proof of Proposition 2.

Proposition 7. Let $\alpha(1), \ldots, \alpha(m)$ be weakly decreasing sequences of $n$ real numbers. There are Hermitian $n$ by $n$ matrices $A(1), \ldots, A(m)$, with $\alpha(s)$ the eigenvalues of $A(s)$, and $\sum_{s=1}^{m} A(s)$ a scalar matrix, if and only if $\left(*_{\mathcal{I}}\right)$ holds for every $\mathcal{I}$ in $S_{r}^{n}(m)$, for all $r<n$.

The implication $\Rightarrow$ is proved as in Proposition 2, but simpler: If $\sum_{s} A(s)=c$, and $\prod_{s} \omega_{I(s)} \neq 0$, there is some subspace $L$ in $\bigcap_{s} \Omega_{I(s)}^{\circ}\left(F_{\bullet}(s)\right)$. By Proposition 1 ,

$$
\sum_{s=1}^{m} \sum_{i \in I(s)} \alpha_{i}(s) \leq \sum_{s=1}^{m} R_{A(s)}(L)=R_{c}(L)=c \cdot r,
$$

and $c=\frac{1}{n} \operatorname{Trace}\left(\sum_{s} A(s)\right)=\frac{1}{n} \sum_{s} \sum_{i=1}^{n} \alpha_{i}(s)$. This proves that $\left(*_{\mathcal{I}}\right)$ holds.

For the converse, first one checks that the region defined by the inequalities $\left(*_{\mathcal{I}}\right)$ and the inequalities that make each of $\alpha(s)$ weakly decreasing has a nonempty interior ( $\mathrm{Fu} 3$, Lemma 2). By a continuity argument, using the compactness of the unitary group $U(n)$, it suffices to prove the existence of the Hermitian matrices when each $\alpha(s)$ consists of $n$ distinct rational numbers, and all of the inequalities $\left(*_{\mathcal{I}}\right)$ are strict; therefore (since multiplying matrices by scalars or adding scalar matrices doesn't change the situation), we may assume that each $\alpha(s)$ consists of $n$ distinct nonnegative integers. Take general flags $F_{\bullet}(s), 1 \leq s \leq m$, and use the partitions $\alpha(s)$ to construct filtrations $V \cdot(s)$. We have seen that this $m$-filtration is stable. By Proposition 6, there is a Hermitian metric on $V$ so that the sum of the projections on the spaces $V^{p}(s), p \geq 1,1 \leq s \leq m$, is a scalar. The conclusion follows from Lemma 1.

Special cases of the following proposition were stated by V. B. Lidskii [L1] and proved by Horn [H2. In fact, Horn used only calculus to show that, assuming that the eigenvalues are distinct, if one is on a boundary of the region of possible eigenvalues for real symmetric matrices, there must be an invariant subspace. This was an early step toward his conjecture.

Proposition 8. Let $A(s)$ be Hermitian $n$ by $n$ matrices, with eigenvalues $\alpha(s)$, for $1 \leq s \leq m$, whose sum $\sum_{s} A(s)$ is a scalar. Suppose that there is some $\mathcal{I}$ in $S_{r}^{n}(m)$ such that $\left(*_{\mathcal{I}}\right)$ holds with equality. Then there is an $r$-dimensional subspace $L$ of $\mathbb{C}^{n}$ that is mapped to itself by each $A(s)$.

To prove this, take a flag $F_{\bullet}(s)=F_{.}(A(s))$ corresponding to $A(s)$ as in Proposition 1. Let $c=\frac{1}{n} \sum_{s} \sum_{i} \alpha_{i}(s)$ be the scalar that the matrices $A(s)$ sum to. Since $\mathcal{I}$ is in $S_{r}^{n}(m)$ there must be an $r$-dimensional subspace $L$ of $\mathbb{C}^{n}$ in $\bigcap_{s} \Omega_{I(s)}\left(F_{\bullet}(s)\right)$. By Proposition 1,

$$
\frac{1}{r} \sum_{s} \sum_{i \in I(s)} \alpha_{i}(s) \leq \frac{1}{r} \sum_{s} R_{A(s)}(L)=\frac{1}{r} R_{\sum_{s} A(s)}(L)=c .
$$

Since $\left(*_{\mathcal{I}}\right)$ holds with equality, we must have $\sum_{i \in I(s)} \alpha_{i}(s)=R_{A(s)}(L)$ for every $s$. By Corollary 1 to Proposition 1, each $A(s)$ must map $L$ to itself. 
Since each $A(s)$ is Hermitian, it follows that it also maps $L^{\perp}$ to itself. The sum of the restrictions of $A(s)$ to $L$ (or $L^{\perp}$ ) is the same scalar $c$, so the process can be repeated for these: either all the inequalities for them are strict, or they can be further decomposed. (This is essentially Totaro's proof [T] of Proposition 6 above.) Proposition 8 can also be proved from properties of moment maps, as in $[\mathrm{Kn}$.

Corollary. Suppose in addition that each $A(s)$ is a real symmetric matrix, and $\prod_{s} \omega_{I(s)}$ is an odd multiple of $\omega_{\{1, \ldots, r\}}$, where $r$ is the cardinality of each $I(s)$. Then there is an $r$-dimensional subspace $L$ of $\mathbb{R}^{n}$ that is preserved by each $A(s)$.

Indeed, the fact that $\prod_{s} \omega_{I(s)}$ is an odd multiple of $\omega_{\{1, \ldots, r\}}$ guarantees that the intersection $\bigcap_{s} \Omega_{I(s)}\left(F_{.}(s)\right)$ of real Schubert varieties must contain a real point. This is obvious if the flags are in general position, since the complex points occur in pairs. It is also true for arbitrary flags, by the results of [Fu1], Chapter 13.

This explains Example 1 in Section 1, which presents real symmetric matrices for which an equality is satisfied but for which there is no real subspace preserved by the matrices. In the present terminology, each $I(s)=\{2,4,6\}$, and $\prod_{s} \omega_{I(s)}=$ $2 \omega_{\{1, \ldots, r\}}$; the corresponding intersection of three real Schubert varieties has two complex points, but no real points. See Sottile's article $\mathrm{So}$ ] for general results about real Schubert calculus.

If each $A(s)$ is real symmetric, if at least one has $n$ distinct eigenvalues, and if some $\left(*_{\mathcal{I}}\right)$ holds with equality, then there is a real subspace of dimension $r$ preserved by each of the $A(s)$. Indeed, the fact that the eigenvalues of at least one $A(s)$ are distinct guarantees (by Proposition 10 below) that $\prod_{s=1}^{m} \omega_{I(s)}=\omega_{\{1, \ldots, r\}}$, so the corollary applies.

It is not hard to see that, in the list of inequalities $\left(*_{\mathcal{I}}\right)$, those for which the sum of the codimensions of the $\omega_{I(s)}$ is less than $r(n-r)$ follow from those where the sum of the codimensions is equal to $r(n-r)$. The latter are the classes for which $\prod_{s} \omega_{I(s)}$ is a nonzero multiple of the class $\omega_{\{1, \ldots, r\}}$ of a point. This is a consequence of Pieri's formula for multiplying a Schubert class by the class $\sigma_{(1)}$ of a hyperplane. This also follows from the stronger results below.

C. Woodward was the first to discover that this reduced list of inequalities $\left(*_{\mathcal{I}}\right)$ is still redundant, by finding the example (12). These examples involve Schubert classes whose intersection number is greater than 1. P. Belkale conjectured and proved that all such inequalities must be redundant. To state his theorem, let $R_{r}^{n}(m)$ be the set of $m$-tuples $\mathcal{I}$ of subsets of $\{1, \ldots, n\}$ of cardinality $r$ such that $\prod_{s} \omega_{I(s)}=\omega_{\{1, \ldots, r\}}$.

Proposition 9. On the space of $m$-tuples $\alpha(1), \ldots, \alpha(m)$ of weakly decreasing sequences of $n$ real numbers, the inequalities $\left(*_{\mathcal{I}}\right)$ for all $\mathcal{I}$ in $S_{r}^{n}(m)$, and all $r<n$, are implied by those in $R_{r}^{n}(m)$ for all $r<n$.

In fact, Knutson and Woodward proved that the extra inequalities are redundant in a particularly strong way, as we had observed in examples:

Proposition 10. Let $\alpha(1), \ldots, \alpha(m)$ be weakly decreasing sequences of n real numbers, satisfying the inequalities $\left(*_{\mathcal{I}}\right)$ for $\mathcal{I}$ in $R_{r}^{n}(m)$, all $r<n$. Suppose, for some $r<n$ and some $\mathcal{I}$ in $S_{r}^{n}(m) \backslash R_{r}^{n}(m)$, we have the reverse inequality

$$
\frac{1}{r} \sum_{s=1}^{m} \sum_{i \in I(s)} \alpha_{i}(s) \geq \frac{1}{n} \sum_{s=1}^{m} \sum_{i=1}^{n} \alpha_{i}(s) .
$$


Then this inequality is actually an equality. Moreover, for every $s$ between 1 and $m$, at least one of the inequalities $\alpha_{1}(s) \geq \alpha_{2}(s) \geq \ldots \geq \alpha_{n}(s)$ must be an equality.

The proofs of Belkale and Woodward follow an idea familiar in the study of stability of vector bundles: a maximal destabilizing subbundle with maximal slope must be unique. As before, take general complete flags $F_{.}(s)$ in $V=\mathbb{C}^{n}$. For any $r$-dimensional subspace $L$ of $V$, there are unique subsets $I(s)$ of $\{1, \ldots, n\}$ of cardinality $r$ such that $L$ is in the intersection $\bigcap_{s=1}^{m} \Omega_{I(s)}^{\circ}\left(F_{\bullet}(s)\right)$. Let $\mathcal{I}(L)=$ $(I(1), \ldots, I(m))$ be this $m$-tuple of subsets. Note that, since the flags are general, this intersection of Schubert cells is nonempty if and only if the corresponding product $\prod_{s=1}^{m} \omega_{I(s)}$ of Schubert classes is not zero. Define the slope of $L, \mu(L)$, by the formula

$$
\mu(L)=\frac{1}{r} \sum_{s=1}^{m} \sum_{i \in I(s)} \alpha_{i}(s)
$$

Suppose $\mathcal{I}$ is in $S_{r}^{n}(m) \backslash R_{r}^{n}(m)$ and $\frac{1}{r} \sum_{s=1}^{m} \sum_{i \in I(s)} \alpha_{i}(s)>\frac{1}{n} \sum_{s=1}^{m} \sum_{i=1}^{n} \alpha_{i}(s)$. There must be some $L$ in the intersection $\bigcap_{s=1}^{m} \Omega_{I(s)}^{\circ}\left(F_{\bullet}(s)\right)$, and $\mu(L)>\mu(V)$ for any such $L$. Let $\mu$ be the maximum of all slopes of all subspaces $L$, and take $r$ maximal such that some $L$ of dimension $r$ has slope $\mu$. Choose a subspace $L$ of dimension $r$ with $\mu(L)=\mu$, and set $\mathcal{I}=\mathcal{I}(L)$. Since $\mu(L)>\mu(V)$, $\mathcal{I}$ is not in $R_{r}^{n}(m)$, so the intersection $\bigcap_{s=1}^{m} \Omega_{I(s)}^{\circ}\left(F_{\bullet}(s)\right)$ has cardinality greater than 1. Let $L^{\prime} \neq L$ be another space in the same intersection. Consider the sum $L+L^{\prime}$, of some dimension $r+t>r$, and set $\mathcal{K}=\mathcal{I}\left(L+L^{\prime}\right)$. The intersection $L \cap L^{\prime}$ has dimension $r-t ;$ set $\mathcal{J}=\mathcal{I}\left(L \cap L^{\prime}\right)$.

The linear algebra fact that, for all $i$ and $s$,

$$
\operatorname{dim}\left(\left(L+L^{\prime}\right) \cap F_{i}(s)\right) \geq \operatorname{dim}\left(L \cap F_{i}(s)\right)+\operatorname{dim}\left(L^{\prime} \cap F_{i}(s)\right)-\operatorname{dim}\left(\left(L \cap L^{\prime}\right) \cap F_{i}(s)\right),
$$

together with equation (31), implies that

$$
\sum_{k \in K(s)} \alpha_{k}(s) \geq \sum_{i \in I(s)} \alpha_{i}(s)+\sum_{i \in I(s)} \alpha_{i}(s)-\sum_{j \in J(s)} \alpha_{j}(s) .
$$

Since $r \cdot \mu(L)=\sum_{s} \sum_{i \in I(s)} \alpha_{i}(s)$, and similarly for the others, this implies the inequality

$$
(r+t) \cdot \mu\left(L+L^{\prime}\right) \geq r \cdot \mu(L)+r \cdot \mu(L)-(r-t) \cdot \mu\left(L \cap L^{\prime}\right) .
$$

Since $\mu(L)=\mu$ and $\mu\left(L \cap L^{\prime}\right) \leq \mu$, the right side is at least $2 r \cdot \mu-(r-t) \cdot \mu=(r+t) \cdot \mu$. Hence $\mu\left(L+L^{\prime}\right) \geq \mu$, which violates the maximality of $r$. See $\mathrm{Gr}$ ] for more about maximal destabilizing subspaces.

For the last assertion in Proposition 10, suppose that all the inequalities $(* \mathcal{I})$ hold for $\mathcal{I}$ in $S_{r}^{n}(m)$, but that there is some $\mathcal{I}$ in $S_{r}^{n}(m) \backslash R_{r}^{n}(m)$ for which equality holds. Suppose for some $s$ that the eigenvalues $\alpha_{1}(s), \ldots, \alpha_{n}(s)$ are all distinct. We know that, for an appropriate metric on $V=\mathbb{C}^{n}$, there are Hermitian operators $A(s)$ whose sum is scalar and so that each of the general flags $F_{\bullet}(s)$ is a flag $F_{\bullet}(A(s))$ corresponding to $A(s)$. Since $\mathcal{I}$ is not in $R_{r}^{n}(m)$, there are at least two $r$-dimensional linear subspaces in $\bigcap_{s=1}^{m} \Omega_{I(s)}\left(F_{\bullet}(s)\right)$. But now for any $L$ in $\bigcap_{s=1}^{m} \Omega_{I(s)}\left(F_{\bullet}(s)\right)$, by 
Proposition 1,

$$
\begin{aligned}
\frac{1}{r} \sum_{s=1}^{m} \sum_{i \in I(s)} \alpha_{i}(s) & \leq \frac{1}{r} \sum_{s=1}^{m} R_{A(s)}(L) \\
& =\frac{1}{r} R_{\sum A(s)}(L)=\frac{1}{n} \sum_{s=1}^{m} \sum_{i=1}^{n} \alpha_{i}(s) .
\end{aligned}
$$

Since the first and last terms are equal, we must have $\sum_{i \in I(s)} \alpha_{i}(s)=R_{A(s)}(L)$ for each $s$. But Corollary 1 to Proposition 1 implies that, if $\alpha(s)$ consists of $n$ distinct eigenvalues, $L$ is unique: there cannot be two or more such $L$ in this intersection. 4

This argument applies also to the case where the intersection of the Schubert classes is not zero or a multiple of the class of a point, for in this case the corresponding intersection of Schubert varieties would be infinite.

There is another fundamental fact from geometric invariant theory that Klyachko uses to make the link between stability and highest weights. Namely, if $\mathcal{L}$ is the restriction to $X$ of the standard line bundle $\mathcal{O}(1)$ on the projective space $\mathbb{P}(E)=G r(1, E)$, a point $x$ is semistable exactly when some positive power $\mathcal{L}^{\otimes N}$ has a section that is invariant by $S L(V)$ and does not vanish at $x$. If each $\alpha(s)$ consists of nonnegative integers, the space of sections $\Gamma\left(X, \mathcal{L}^{\otimes N}\right)$ is isomorphic to the dual of the tensor product $\otimes_{s=1}^{m} V(N \alpha(s))$ of representations with highest weights $N \alpha(s)$, where, for any $n$-tuple $\alpha=\left(\alpha_{1}, \ldots, \alpha_{n}\right), N \alpha$ denotes $\left(N \alpha_{1}, \ldots, N \alpha_{n}\right)$. The bundle $\mathcal{L}^{\otimes N}$ has a nonzero $S L(V)$-invariant section if and only if the representation $\otimes_{s} V(N \alpha(s))$ contains the trivial representation of $S L(V)$. The conditions $\left(*_{\mathcal{I}}\right)$ imply that a general point $x$ in $X$ is semistable, and therefore $\otimes_{s} V(N \alpha(s))$ must contain this trivial representation. (For more detail see [Fu3]). This proves

Proposition 11. Suppose $\alpha(1), \ldots, \alpha(s)$ are partitions of length at most $n$, and $\left(*_{\mathcal{I}}\right)$ holds whenever $\prod_{s} \omega_{I(s)}=\omega_{\{1, \ldots, r\}}$. Then there is some positive integer $N$ such that $\otimes_{s} V(N \alpha(s))$ contains the trivial representation of $S L(V)$.

\section{CoAdjoint orbits And MOMEnT MAPS}

The methods of coadjoint orbits and moment maps from symplectic geometry are powerful tools for studying many of the problems discussed here. A full discussion would go beyond the scope of this article. Here we sketch how this approach can be used to simplify and unify some of the results obtained in Section 7. An excellent account of this method can be found in $[\mathrm{Kn}]$.

Let $H$ be the space of Hermitian $n$ by $n$ matrices. For $\alpha$ a weakly decreasing sequence of $n$ real numbers, let $O_{\alpha} \subset H$ be the set of Hermitian matrices with spectrum $\alpha$. This is an orbit of the (compact) unitary group $U(n)$ on $H$, acting by conjugation. The Lie algebra of $U(n)$ is the space $\mathfrak{u}(n)$ of skew-symmetric matrices. Identify $H$ with the (real) dual space $\mathfrak{u}(n)^{*}$ by the map which takes $A$ in

\footnotetext{
${ }^{4}$ One can also prove this last part the same way as the first: If $\left(*_{\mathcal{I}}\right)$ holds with equality, but there are two spaces $L$ and $L^{\prime}$ in $\bigcap_{s=1}^{m} \Omega_{I(s)}^{\circ}\left(F_{\bullet}(s)\right)$, the same argument shows that

$$
\operatorname{dim}\left(\left(L+L^{\prime}\right) \cap F_{i}(s)\right)=\operatorname{dim}\left(L \cap F_{i}(s)\right)+\operatorname{dim}\left(L^{\prime} \cap F_{i}(s)\right)-\operatorname{dim}\left(\left(L \cap L^{\prime}\right) \cap F_{i}(s)\right)
$$

for any $s$ and $i$ such that $\alpha_{i}(s)-\alpha_{i+1}(s)>0$. If there is an $s$ with all $\alpha_{i}(s)$ distinct, the display must be an equality for all $i$. This implies, by an easy induction on $i$, that $L \cap F_{i}(s)=L^{\prime} \cap F_{i}(s)$ for all $i$, so $L=L^{\prime}$.
} 
$H$ to $[B \mapsto-\operatorname{tr}(i A \cdot B)]$ in $\mathfrak{u}(n)^{*}$. This identifies $O_{\alpha}$ with an orbit in $\mathfrak{u}(n)^{*}$, i.e., with a coadjoint orbit.

If each $\alpha_{i}$ is integral, $O_{\alpha}$ can be identified with a partial flag variety $F l(\alpha)$, which has subspaces of dimensions $i$ for each $i$ such that $\alpha_{i}>\alpha_{i+1}$. Corresponding to $A$ in $O_{\alpha}$ one has the flag of sums of eigenspaces corresponding to $\alpha_{1}, \ldots, \alpha_{i}$, for $1 \leq i \leq n$. As in Section 7, there is a natural embedding of $F l(\alpha)$ in $\mathbb{P}(V(\alpha))$.

Every coadjoint orbit has a natural symplectic structure, as does every submanifold of projective space. It is a basic fact that these symplectic structures agree on $O_{\alpha} \cong F l(\alpha) \subset \mathbb{P}(V(\alpha))$.

Now suppose $\alpha(1), \ldots, \alpha(m)$ are given, each integral, with $\sum_{s} \sum_{i} \alpha_{i}(s)=0$. We have closed embeddings

$$
X=\prod_{s=1}^{m} O_{\alpha(s)}=\prod_{s=1}^{m} F l(\alpha(s)) \subset \prod_{s=1}^{m} \mathbb{P}(V(\alpha(s))) \subset \mathbb{P}\left(\otimes_{s=1}^{m} V(\alpha(s))\right) .
$$

The diagonal action of $U(n)$ on $X$ is a Hamiltonion action, which determines a moment mapping from $X$ to $\mathfrak{u}(n)^{*}$. Identifying $\mathfrak{u}(n)^{*}$ with $H$, this moment mapping

$$
\mu: X \rightarrow H
$$

is simply addition: $\mu(A(1), \ldots, A(m))=A(1)+\ldots+A(m)$.

A basic result of symplectic geometry - inspired, interestingly, by A. Horn's other paper $\mathrm{H1}$ about eigenvalues of Hermitian matrices - is that the intersection of $\mu(X)$ with a Weyl chamber is a convex polytope. Here it says that the possible eigenvalues $\gamma=\left(\gamma_{1} \geq \ldots \geq \gamma_{n}\right)$ of a sum $\sum A(s)$ form a convex polytope.

In connection with the moment map $\mu$, one has the symplectic reduction $\mu^{-1}(0) / U(n)$. This space is in fact homeomorphic to the quotient space of $X$ by $G=G L(n, \mathbb{C})$ constructed by geometric invariant theory $([\mathrm{MFK}], \S 8)$ :

$$
\mu^{-1}(0) / U(n) \cong X^{s s} / G \cong \operatorname{Proj}(R),
$$

where $R=\oplus R_{N}, R_{N}=\Gamma(X, \mathcal{O}(N))^{G}=\left(\otimes V(N \alpha(s))^{*}\right)^{G}$. From this one sees the equivalence of the following three conditions, which say that each of the three spaces in (33) is nonempty:

(i) there are Hermitian $A(1), \ldots, A(m)$ with eigenvalues $\alpha(1), \ldots, \alpha(m)$ such that $\sum_{s} A(s)=0$;

(ii) a general point (or some point) in $X=\prod F l(\alpha(s))$ is semistable;

(iii) for infinitely many (or one) $N \geq 1, \otimes_{s} V(N \alpha(s))^{G} \neq 0$.

As in Section 7 (cf. [Fu3]), (ii) is equivalent to the condition that for general flags $F_{\bullet}(1), \ldots F_{\bullet}(m)$ and all subspaces $L$ of $\mathbb{C}^{n}$,

$$
\sum_{s=1}^{m} \sum_{i=1}^{n} \alpha_{i}(s) \operatorname{dim}\left(L \cap F_{i}(s) / L \cap F_{i-1}(s)\right) \leq 0
$$

If $I(s)=\left\{i \in\{1, \ldots, n\} \mid L \cap F_{i}(s) \neq L \cap F_{i-1}(s)\right\}$, this says that $L$ is in $\bigcap_{s} \Omega_{I(s)}^{\circ} F_{\bullet}(s)$. Since the flags are general position, (ii) is equivalent to

(ii') $\sum_{s} \sum_{i \in I(s)} \alpha_{i}(s) \leq 0$ whenever $\prod_{s} \omega_{I(s)} \neq 0$.

The equivalence of (i), (ii'), and (iii) re-proves Propositions 5, 7, and 11. For generalizations and variations, see $[\mathrm{AW}], \mathrm{BS},[\mathrm{DRW}], \mathrm{He},[\mathrm{OS},[\mathrm{SQS}], \mathrm{Ta}]$. 


\section{SATURATion}

Proposition 11 is close to solving the highest weight problem, except for the serious possibility that the integer $N$ that appears might be greater than 1 . In fact, for representations of some classical groups, such integers are necessary. For example, J. Stembridge points out that if $\omega$ is the highest weight of the basic spin representation $V(\omega)$ of $\operatorname{Spin}_{9}(\mathbb{C})$, then $V(\omega) \otimes V(\omega)$ decomposes into four irreducible representations, while $V(2 \omega) \otimes V(2 \omega)$ contains six irreducible representations of the form $V(2 \eta)$ for some weights $\eta$. Similarly in Schubert calculus, P. Pragacz points out that for the Lagrangian Grassmannian of 3-planes in 6-space, $\sigma_{642}$ occurs in $\sigma_{42} \cdot \sigma_{42}$, although $\sigma_{321}$ does not occur in $\sigma_{21} \cdot \sigma_{2,1}$; cf. [PR].

The key to this is provided by the following result of A. Knutson and T. Tao, which solves what is called the Saturation Problem.

Proposition 12 ([KT] $)$. If $\alpha, \beta$, and $\gamma$ are a triple of partitions, and $c_{N \alpha N \beta}^{N \gamma} \neq 0$, then $c_{\alpha \beta}^{\gamma} \neq 0$.

Their proof uses a wonderful new combinatorial description of these LittlewoodRichardson numbers as the number of geometric figures called "honeycombs" satisfying some conditions. Their original proof also used another new description that they call "hives". Buch $[\mathrm{Bu}]$ has given a shorter version of their proof, entirely in the language of hives. For more about this Saturation Problem (before it was solved), see A. Zelevinsky's article [Z]. Recently H. Derksen and J. Weyman DW] have given a different proof of Proposition 12, using representations of quivers. It is interesting to note that Johnson's unpublished thesis [J] contains a solution of the saturation problem when the partitions have lengths at most four.

A. Postnikov and Zelevinsky have pointed out how this result is equivalent to one that looks stronger:

Proposition 13. If $\alpha(1), \ldots, \alpha(m)$ and $\beta$ are partitions of length at most $n$, and $N$ is a positive integer such that $V(N \beta)$ occurs in the decomposition of $\otimes_{s} V(N \alpha(s))$ as representations of $G L_{n}(\mathbb{C})$, then $V(\beta)$ occurs in the decomposition of $\otimes_{s} V(\alpha(s))$.

This follows from that fact that the number of times a representation $V(\beta)$ occurs in the decomposition of $\otimes_{s} V(\alpha(s))$ is equal to a Littlewood-Richardson coefficient $c_{\alpha \beta}^{\gamma}$, where $\alpha$ and $\gamma$ are obtained as follows. Arrange the Young diagrams of $\alpha(1), \alpha(2), \ldots, \alpha(m)$ corner to corner from lower left to upper right, so that the upper right corner of the diagram of $\alpha(i)$ just touches the lower left corner of $\alpha(i+1)$; these form a skew diagram $\gamma \backslash \alpha$. The reason for the equality is that both numbers are the numbers of skew tableaux on the shape $\gamma \backslash \alpha$ whose rectification is a given tableau of shape $\beta$ (cf. [Fu2], §5.1). Proposition 13 can also be proved directly, using the methods of [KT] or [DW].

Note that the corresponding result follows for representations of $S L_{n}(\mathbb{C})$, since representations of $S L_{n}(\mathbb{C})$ differ from those of $G L_{n}(\mathbb{C})$ only by powers of the determinant representation $\bigwedge^{n} \mathbb{C}^{n}(\mathrm{cf} .[\mathrm{FH}], \S 15)$.

Proposition 14. Let $\alpha(1), \ldots, \alpha(m)$ be partitions of lengths at most $n$, and suppose $c=\frac{1}{n} \sum_{s=1}^{m} \sum \alpha_{i}(s)$ is an integer. Then the representation $\otimes_{s=1}^{m} V(\alpha(s))$ of $G L_{n}(\mathbb{C})$ contains the representation $\left(\bigwedge^{n} \mathbb{C}^{n}\right)^{\otimes c}$ if and only if $\left(*_{\mathcal{I}}\right)$ holds for all $\mathcal{I} \in S_{r}^{n}(m)$ and all $r<n$.

These conditions are equivalent to the condition that the Schur polynomial $s_{\left(c^{n}\right)}$ occurs in the product $\prod_{s=1}^{m} s_{\alpha(s)}$. 
As to geometric invariant theory itself, the ring $\oplus_{N>0} \Gamma\left(X, \mathcal{L}^{\otimes N}\right)^{S L(V)}$ is the homogeneous coordinate ring of the GIT quotient variety $X / / S L(V)$ of $X$ by $S L(V)$; that is, $X / / S L(V)=\operatorname{Proj}(R)$. One may ask whether this graded ring $R$ is generated by its homogeneous part of degree 1 . Unfortunately, this is not always true, even for these special varieties that are products of partial flag varieties:

Example 5. For $n=3$, let $X$ be the Cartesian product of 6 copies of $\mathbb{P}^{2}=\mathbb{P}(V)$, and $\mathcal{L}=\mathcal{O}(1,1,1,1,1,1)$ the tensor product of the pullbacks of the line bundles $\mathcal{O}(1)$ on the factors. The homogeneous part $\Gamma(X, \mathcal{O}(2,2,2,2,2,2))^{S L_{3}(\mathbb{C})}$ of degree 2 has dimension 16 , while that of degree 1 has dimension 5 , and $16>\left(\begin{array}{c}5+1 \\ 2\end{array}\right)$. Equivalently, if $\alpha=(5,4,3,2,1), \beta=(2,2,2)$, and $\gamma=(6,5,4,3,2,1)$, then $c_{\alpha \beta}^{\gamma^{2}}=$ 5 , while $c_{2 \alpha 2 \beta}^{2 \gamma}=16>\left(\begin{array}{c}c_{\alpha \beta}^{\gamma}+1 \\ 2\end{array}\right)$. Buch has verified by computer that the smallest $N \cdot \sum \gamma_{i}$ for which there is an $\alpha, \beta, \gamma$ and $N$ for which $c_{N \alpha N \beta}^{N \gamma}>\left({ }_{\alpha \beta}^{\gamma}+N-1\right)$ is for $N \cdot \sum \gamma_{i}=30$, with $\alpha=(4,3,2,1), \beta=(2,2,1), \gamma=(5,4,3,2,1)$, and $N=2$, where again $c_{\alpha \beta}^{\gamma}=5$, and $c_{2 \alpha 2 \beta}^{2 \gamma}=16$. For a geometric description of $\left(\mathbb{P}^{2}\right)^{6} / / S L(3, \mathbb{C})$, see [DO], §VII.3.

A. Okounkov speculates that the function $f(N)=c_{N \alpha N \beta}^{N \gamma}$ may be log concave, i.e., that

$$
f(N)^{2} \geq f(N-1) \cdot f(N+1)
$$

for all $N \geq 1$.

For a general product $X$ of partial flag varieties, is there a reasonable criterion to tell when this algebra is generated by its part in degree 1 , or to find an $N$ such that the algebra $\oplus_{k \geq 0} \Gamma\left(X, \mathcal{L}^{\otimes k N}\right)^{S L(V)}$ is generated by $\Gamma\left(X, \mathcal{L}^{\otimes N}\right)^{S L(V)}$ ?

Knutson, Tao, and Woodward [KTW] have also announced a proof of a conjecture we had made that $c_{N \alpha N \beta}^{N \gamma}=1$ if and only if $c_{\alpha \beta}^{\gamma}=1$. Buch $\mathrm{Bu}$ deduced from this that, for $n \geq 3$, if each of the partitions in $(\alpha, \beta, \gamma)$ consists of $n$ distinct integers, and if each of the inequalities $\left(*_{I J K}\right)$ holds strictly, then $c_{\alpha \beta}^{\gamma}$ must be at least 2 .

\section{Proofs of the theorems}

Most of the theorems in Sections 1-4 follow easily from the propositions proved in Sections 6,7 , and 9 . Let us start by summarizing the main results, but generalized to an arbitrary number of factors. Alternative versions are stated in brackets.

\subsection{The main theorem.}

Theorem 17. Let $\alpha(1), \ldots, \alpha(m)$ and $\gamma$ be weakly decreasing sequences of $n$ real numbers, with $\sum_{i=1}^{n} \gamma_{i}=\sum_{s=1}^{m} \sum_{i=1}^{n} \alpha_{i}(s)$. The following are equivalent:

(1) $\sum_{k \in K} \gamma_{k} \leq \sum_{s=1}^{m} \sum_{i \in I(s)} \alpha_{i}(s)$ is satisfied for all subsets $I(1), \ldots, I(m), K$ of the same cardinality $r$ of $\{1, \ldots, n\}$ such that $\sigma_{\lambda(K)}$ occurs in $\prod_{s=1}^{m} \sigma_{\lambda(I(s))}$ [with coefficient 1] in $H^{*}\left(G r\left(r, \mathbb{C}^{n}\right)\right)$, and all $r<n$.

(2) $\alpha(1), \ldots, \alpha(m)$ and $\gamma$ are eigenvalues of Hermitian matrices $A(1), \ldots, A(m)$ and $C$, such that $C=\sum_{s=1}^{m} A(s)$.

If each of $\alpha(1), \ldots, \alpha(m)$ and $\gamma$ consists of integers, (1) and (2) are equivalent to

(3) The representation $V(\gamma)$ of $G L_{n}(\mathbb{C})$ occurs in $\otimes_{s=1}^{m} V(\alpha(s))$.

If each of $\alpha(1), \ldots, \alpha(m)$ and $\gamma$ is a partition, these are equivalent to 
(4) For some [every] discrete valuation ring $R$, there exist $n$ by $n$ matrices $A(1)$, $\ldots, A(m)$ and $C$ with invariant factors $\alpha(1), \ldots, \alpha(m)$ and $\gamma$ such that $C=$ $A(1) \cdot \ldots \cdot A(m)$.

If, in addition, $q$ is an integer at least as large as each $\alpha_{1}(s)$ and $\gamma_{1}$, these are equivalent to

(5) The class $\sigma_{\gamma}$ occurs with positive coefficient in the product $\prod_{s=1}^{m} \sigma_{\alpha(s)}$ in $H^{*}\left(G r\left(n, \mathbb{C}^{n+q}\right)\right)$.

We show how to deduce this theorem from the propositions in the preceding sections. As in the proof of Proposition 2, $\sigma_{\lambda(K)}$ occurs in $\prod_{s=1}^{m} \sigma_{\lambda(I(s))}$ [with coefficient 1] if and only if $\prod_{s=1}^{m} \omega_{I(s)^{\prime}} \cdot \omega_{K}$ is a nonzero multiple [equal to 1] of $\omega_{\{1, \ldots, r\}}$. Let $\beta(s)=\left(-\alpha_{n}(s), \ldots,-\alpha_{1}(s)\right)$, for $1 \leq s \leq m$, and let $\beta(m+1)=\gamma$. The condition (2) is equivalent to the existence of Hermitian matrices $B(s)$ with eigenvalues $\beta(s), 1 \leq s \leq m+1$, whose sum is zero (as one sees by setting $A(s)=$ $-B(s)$ and $C=B(m+1))$. From Proposition 7 [and Proposition 9], (2) is equivalent to the inequalities $\sum_{s=1}^{m} \sum_{i \in I(s)^{\prime}} \beta_{i}(s)+\sum_{k \in \mathcal{K}} \gamma_{k} \leq 0$ whenever $\prod_{s=1}^{m} \omega_{I(s)^{\prime}} \cdot \omega_{K}$ is a nonzero multiple [equal to 1] of $\omega_{\{1, \ldots, r\}}$. This proves the equivalence of (1) and (2).

Condition (3) is equivalent to $\otimes_{s=1}^{m} V(\alpha(i))^{*} \otimes V(\gamma)$ containing the trivial representation of $G L_{n}(\mathbb{C})$, where $V(\alpha(s))^{*}$ is the dual representation to $V(\alpha(s))$, which has highest weight $\left(-\alpha_{n}(s), \ldots,-\alpha_{1}(s)\right)$. So the equivalence of (3) with (1) follows from Propositions 11 and 13.

Condition (3) is equivalent to the assertion that the Schur polynomial $s_{\gamma}$ occurs in the product $\prod_{s=1}^{m} s_{\alpha(s)}$. The equivalence of this with (4) is seen by induction on $m$, the case $m=2$ having been seen in Section 6 . Indeed, $s_{\gamma}$ occurs in $\prod_{s=1}^{m} s_{\alpha(s)}$ if and only if there is some $\beta$ such that $s_{\beta}$ occurs in $\prod_{s=1}^{m-1} s_{\alpha(s)}$ and $s_{\gamma}$ occurs in $s_{\beta} \cdot s_{\alpha(m)}$. By induction, the first corresponds to a product $A(1) \cdot \ldots \cdot A(m-1)=B$ with invariant factors $\alpha(1), \ldots, \alpha(m-1)$ and $\beta$, and the second to a product $B \cdot A(m)=C$ with invariant factors $\beta, \alpha(m)$, and $\gamma$.

That (3) and (5) are equivalent follows as before from the fact that there is a homomorphism from the ring of symmetric polynomials onto the cohomology of the Grassmann manifold taking $s_{\alpha}$ to $\sigma_{\alpha}$ for all $\alpha$.

10.2. Horn's conjecture. We turn to the proof of Theorem 12, which implies Horn's conjecture. We start with a general combinatorial lemma.

Lemma 4. (i) Let $I=\left\{i_{1}<\ldots<i_{r}\right\} \subset\{1, \ldots, n\}$, and let $\lambda=\left(i_{r}-r, \ldots, i_{1}-1\right)$ be the corresponding partition. Let $I^{\prime}=\{i \in\{1, \ldots, n\} \mid n+1-i \notin I\}$. Then the partition corresponding to $I^{\prime}$ is the conjugate partition $\lambda^{\prime}$ to $\lambda$.

(ii) If $\lambda, \mu$, and $\nu$ are partitions, with conjugates $\lambda^{\prime}, \mu^{\prime}$, and $\nu^{\prime}$, then $c_{\lambda_{\mu}}^{\nu}=c_{\lambda^{\prime} \mu^{\prime}}^{\nu^{\prime}}$.

The first is an elementary combinatorial fact (cf. [Mac] $\S \mathrm{I}(1.7)$ ) that can be seen visually by identifying $\lambda$ with its Young diagram, which is traced out by a sequence of vertical steps going from lower left to upper right of the $r$ by $n-r$ rectangle of boxes. If $\lambda=\lambda(I)$, then $I$ specifies which of these $n$ steps are vertical, and its complement specifies which are horizontal, so $I^{\prime}$ specifies the vertical steps for $\lambda^{\prime}$. The second assertion is not obvious from the definition of Littlewood-Richardson coefficients, but follows from other descriptions, cf. [Fu2 $\S 5.1$.

Now we prove that $T_{r}^{n}=S_{r}^{n}$ for all $r<n$, by induction on $r$. It follows from the definition that $T_{1}^{n}=S_{1}^{n}=U_{1}^{n}$. Let $(I, J, K)$ be a triple in $U_{r}^{n}$, with $r>1$, and 
let $(\lambda, \mu, \nu)$ be the corresponding triple of partitions, each of length at most $r$. The condition for $(I, J, K)$ to be in $T_{r}^{n}$ is that, for all $p<r$ and all $(F, G, H) \in T_{p}^{r}$,

$$
\sum_{f \in F} i_{f}+\sum_{g \in G} j_{g} \leq \sum_{h \in H} k_{h}+p(p+1) / 2
$$

Since $i_{a}-a=\lambda_{r+1-a}$, and similarly for $J$ and $K$, this is equivalent to the inequality

$$
\sum_{f \in F} \lambda_{r+1-f}+\sum_{g \in G} \mu_{r+1-g} \leq \sum_{h \in H} \nu_{r+1-h} .
$$

The assumption that $(I, J, K)$ is in $U_{r}^{n}$ says that the triple $(\lambda, \mu, \nu)$ satisfies $(*)$, i.e., $\sum \lambda_{i}+\sum \mu_{i}=\sum \nu_{i}$. The preceding inequality is therefore equivalent to

$$
\sum_{f \in F^{\prime}} \lambda_{f}+\sum_{g \in G^{\prime}} \mu_{g} \geq \sum_{h \in H^{\prime}} \nu_{h}
$$

where $F^{\prime}=\{f \in\{1, \ldots, r\} \mid r+1-f \notin F\}$, and $G^{\prime}$ and $H^{\prime}$ defined similarly. By induction, we know that $T_{p}^{r}=S_{p}^{r}$, and by Lemma 4 we know that $(F, G, H)$ is in $S_{p}^{r}$ if and only if $\left(F^{\prime}, G^{\prime}, H^{\prime}\right)$ is in $S_{r-p}^{r}$. Hence the inequalities in question are equivalent to the inequalities

$$
\sum_{h \in H} \nu_{h} \leq \sum_{f \in F} \lambda_{f}+\sum_{g \in G} \mu_{g}
$$

for all $q=r-p<r$ and all $(F, G, H)$ in $S_{q}^{r}$. But by the equivalence of (3) and (5) of Theorem 17 (for the case $m=2$ ), we know that the validity of these inequalities $\left(*_{F G H}\right)$ for all $(F, G, H)$ in $S_{p}^{r}$ for all $p<r$ is equivalent to the assertion that $c_{\lambda \mu}^{\nu}$ is not zero. This means that $(I, J, K)$ is in $S_{r}^{n}$.

The same idea proves the following version for an arbitrary number $m$ of factors, but in a symmetric form where all factors are treated equally (so without a duality argument). Let $U_{r}^{n}(m)$ be the set of $m$-tuples $I(1), \ldots, I(m)$ of subsets of cardinality $r$ in $\{1, \ldots, n\}$ such that

$$
\sum_{s=1}^{m} \sum_{i \in I(s)} i=(m-1) r(n-r)+m r(r+1) / 2 .
$$

Define $S_{r}^{n}(m) \subset U_{r}^{n}(m)$ to be those $m$-tuples such that the product $\prod \omega_{I(s)}$ of the corresponding Schubert varieties is a nonzero multiple of the class of a point in $\operatorname{Gr}\left(r, \mathbb{C}^{n}\right)$, as in Section 6. Define $T_{r}^{n}(m) \subset U_{r}^{n}(m)$ inductively to be the set such that, for all $p<r$ and all $\mathcal{F}=(F(1), \ldots, F(m))$ in $T_{p}^{r}(m)$,

$$
\sum_{s=1}^{m} \sum_{f \in F(s)} i_{f}(s) \geq(m-1) p(n-r)+m p(p+1) / 2 .
$$

Theorem 18. $S_{r}^{n}(m)=T_{r}^{n}(m)$ for all positive integers $m, r$, and $n$ with $r<n$.

This is easier to prove. For $r=1, T_{1}^{n}(m)=S_{1}^{n}(m)=U_{1}^{n}(m)$. For $r>1$, let $\mathcal{I}=(I(1), \ldots, I(m))$ be in $U_{r}^{n}(m)$, and define $\lambda(s)$ so that $\sigma_{\lambda(s)}=\omega_{I(s)}$, i.e., $i_{j}(s)=n-r+j-\lambda_{j}(s)$ for $1 \leq j \leq r$. Then $\mathcal{I}$ is in $T_{r}^{n}(m)$ if and only if $\sum_{s} \sum_{f \in F(s)}\left(i_{f}(s)-f\right) \geq(m-1) p(n-r)$ for all $\mathcal{F}$ in $T_{p}^{r}(m)$ and all $p<r$. This is equivalent to the inequality $\sum_{s} \sum_{f \in F(s)} \lambda_{f}(s) \leq p(n-r)$, or $\frac{1}{p} \sum_{s} \sum_{f \in F(s)} \lambda_{f}(s) \leq$ $n-r$ for all $\mathcal{F}$ in $T_{p}^{r}(m)$ and all $p<r$. By Proposition 14, and the inductive fact that $T_{p}^{r}(m)=S_{p}^{r}(m)$, this is equivalent to saying that $\prod_{s} \sigma_{\lambda(s)}=d \sigma_{\rho}$ for some $d \neq 0$, where $\rho=(n-r)^{r}$. This says that $\mathcal{I}$ is in $S_{r}^{n}(m)$. 
By proving Theorems 17 and 18, we have completed the proofs of Theorems 1 and 2 in their general forms for an arbitrary number of factors. Theorem 5 , also for any number of factors, follows similarly from Proposition 8.

10.3. Compact operators. To prove Theorem 6 , for any number of compact self-adjoint operators $A(1), \ldots, A(m)$ and $C=\sum_{s=1}^{m} A(s)$ on a Hilbert space $H$, we want to show that all the inequalities of (1) of Theorem 17 are valid. Choose a finite-dimensional subspace $V$ of $H$ that contains eigenvectors corresponding to the $n$ largest eigenvalues of each of the operators under consideration. It then suffices to apply the finite-dimensional result to the operators $A(1)_{V}, \ldots, A(m)_{V}$, and $C_{V}$; here, for any operator $A, A_{V}$ denotes the composite $V \rightarrow H \rightarrow H \rightarrow V$, where the first map is the inclusion, the second is $A$, and the third is orthogonal projection. The same argument as in Corollary 2 to Proposition 1 shows that the $k^{\text {th }}$ largest eigenvalue of $A_{V}$ is no larger than the $k^{\text {th }}$ largest eigenvalue of $A$. The operators $A(s)_{V}$ and $C_{V}$ will therefore have the same $n$ largest eigenvalues as $A(s)$ and $C$. For more on eigenvalues of compact operators, see $[\mathrm{W},[\mathrm{Zw}],[\mathrm{Fr}]$, and $[\mathrm{R}]$.

10.4. Invariant factors. We have seen the general version of Theorem 7 in Theorem 17. Theorem 8 follows from Theorem 7 by localization. This is easiest to see in the version about short exact sequences of modules of finite length. To specify such an exact sequence over a principal ideal domain $R$ is equivalent to specifying a short exact sequence of modules of finite length over a finite number of localizations $R_{\mathfrak{p}}$ of $R$, each of which is a discrete valuation ring.

Theorem 9 follows easily from Theorem 8. Indeed, given $C$ (i.e., $X$ ), there is an exact sequence

$$
0 \rightarrow \mathcal{A} \rightarrow \mathcal{C} \rightarrow \mathcal{B} \rightarrow 0
$$

where $\mathcal{A}, \mathcal{B}$, and $\mathcal{C}$ are the cokernels of $A, B$, and $C$, so the necessity follows from Theorem 8. Conversely, given $A$ and $B$ with invariants $a_{1} \subset \ldots \subset a_{p}$ and $b_{1} \subset \ldots \subset b_{q}$, and possible invariants $c_{1} \subset \ldots \subset c_{n}$ satisfying the conditions displayed in Theorem 9, Theorem 8 implies that there is such an exact sequence, with $\mathcal{A}$ and $\mathcal{B}$ the cokernels of $A$ and $B$. The standard argument used to resolve a short exact sequence of modules by a long exact sequence of free modules says that the dotted arrows in the following diagram can be filled in:

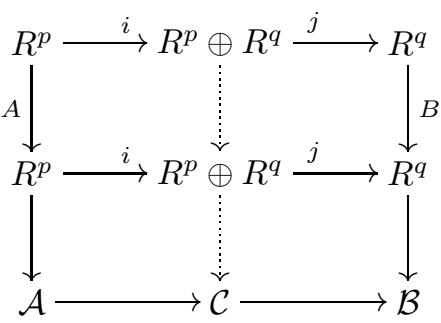

Here $i$ and $j$ are the canonical embeddings and projections: $i(v)=(v, 0), j(v, w)=$ $w$. This produces a matrix $C$ of the required form with cokernel $\mathcal{C}$.

To deduce Theorem 10 from Theorem 9, we use the standard fact that an endomorphism of a finite dimensional vector space over a field $F$, up to similarity, is the same as a finitely generated torsion module over $R=F[T]$, up to isomorphism. The invariant factors are the same for the endomorphism and the $R$-module, and they determine the endomorphism up to similarity. 
10.5. Highest weights. Theorems 11 and 14 are special cases of Theorem 17 together with Theorem 12. Theorem 13 follows from Proposition 9, together with the fact that $(I, J, K)$ is in $S_{r}^{n}$ (resp. $\left.R_{r}^{n}\right)$ if and only if $\left(I^{\prime}, J^{\prime}, K\right)$ is in $S_{r}^{n}(3)$ (resp. $\left.R_{r}^{n}(3)\right)$. Here $R_{r}^{n}(m)$ is defined to be the subset of $S_{r}^{n}(m)$ such that the product $\prod \omega_{I(s)}$ is equal to the class of a point.

10.6. Singular values. We next prove the theorems about singular values. For the additive version in Theorem 15, the fact that all the inequalities $\left(*^{*} I_{I J K}\right)$ must be satisfied follows immediately from Theorem 1 , since if $C=A+B$ is a sum of arbitrary matrices, then

$$
\left(\begin{array}{cc}
0 & C \\
C^{*} & 0
\end{array}\right)=\left(\begin{array}{cc}
0 & A \\
A^{*} & 0
\end{array}\right)+\left(\begin{array}{cc}
0 & B \\
B^{*} & 0
\end{array}\right)
$$

is a sum of Hermitian matrices. (In fact, if one uses the set $S_{r}^{n}$ instead of the set $T_{r}^{n}$, the necessity follows from the simple Proposition 2.) The full theorem was recently proved by O'Shea and Sjamaar [OS], $\S 9$ (also in terms of the sets $S_{r}^{n}$ ), using moment maps, although they do not mention singular values. Unlike the other theorems described here, we do not know another proof for this converse. The same arguments prove the generalization of Theorem 15 to any number of factors.

The easy half of the multiplicative version (Theorem 16) can be proved by using a variation of the Hersch-Zwahlen lemma, which is based on an idea of Amir-Moéz [AM], cf. [TT1. First we require a multiplicative analogue of the Rayleigh trace. Suppose $A$ is a positive semidefinite Hermitian $n$ by $n$ matrix. Let $\alpha_{1} \geq \ldots \geq \alpha_{n} \geq 0$ be the eigenvalues of $A$, and let $v_{1}, \ldots, v_{n}$ be corresponding orthonormal eigenvectors. Let $F_{\text {. }}(A)$ be the flag with $F_{k}(A)$ spanned by $v_{1}, \ldots, v_{k}$. If $L \subset \mathbb{C}^{n}$ is an $r$-dimensional subspace, define $D_{A}(L)$ to be the determinant of the matrix $\left(A u_{i}, u_{j}\right)_{1 \leq i, j \leq r}$, where $u_{1}, \ldots, u_{r}$ is an orthonormal basis of $L$. Equivalently, $D_{A}(L)$ is the determinant of the mapping $A_{L}$ of Corollary 3 to Proposition 1.

Proposition 15. For any subset $I \subset\{1, \ldots, n\}$ of cardinality $r$,

$$
\prod_{i \in I} \alpha_{i}=\operatorname{Min}_{L \in \Omega_{I}\left(F_{\bullet}(A)\right)} D_{A}(L) .
$$

To prove this, write $u_{i}=\sum_{k=1}^{n} x_{i k} v_{k}$. Then $\left(A u_{i}, u_{j}\right)$ is the $i, j$ entry of the matrix $X D X^{*}$, where $X$ is the $r$ by $n$ matrix $\left(x_{i j}\right)$, and $D$ is the diagonal matrix with diagonal entries $\alpha_{1}, \ldots, \alpha_{n}$. Therefore

$$
D_{A}(L)=\sum_{J} \operatorname{det}\left(X^{J}\right) \cdot \prod_{j \in J} \alpha_{j} \cdot \operatorname{det}\left(X_{J}^{*}\right)=\sum_{J}\left|\operatorname{det}\left(X^{J}\right)\right|^{2} \cdot \prod_{j \in J} \alpha_{j},
$$

where the sum is over all subsets $J$ of $\{1, \ldots, n\}$ of cardinality $r, X^{J}$ is the submatrix of $X$ with columns labeled by $J$, and $X_{J}^{*}$ is the submatrix of $X^{*}$ with rows labeled by $J$. Take $u_{p}$ in $L \cap F_{i_{p}}$ successively as in the proof of Proposition 1 . The $p^{\text {th }}$ row of the matrix $X$ ends in column $i_{p}$, so $\operatorname{det}\left(X^{J}\right)=0$ unless $j_{p} \leq i_{p}$ for all $p$. For such $J, \prod_{j \in J} \alpha_{j} \geq \prod_{i \in I} \alpha_{i}$. Moreover, $\sum_{J}\left|\operatorname{det}\left(X^{J}\right)\right|^{2} \geq \operatorname{det}\left(X X^{*}\right)=1$. These inequalities imply that $D_{A}(L) \geq \prod_{i \in I} \alpha_{i}$. As before, equality is achieved by taking $L$ to be spanned by those vectors $v_{i}$ for $i$ in $I$. 
Proposition 16. Let $C=A(1) \cdot \ldots \cdot A(m)$ be a product of $m$ arbitrary $n$ by $n$ matrices. Suppose $L_{1}, \ldots, L_{m}$ are $r$-dimensional subspaces of $\mathbb{C}^{n}$ with $A(s)\left(L_{s}\right) \subset$ $L_{s-1}$ for $2 \leq s \leq m$. Then

$$
D_{C^{*} C}\left(L_{m}\right)=\prod_{s=1}^{m} D_{A(s)^{*} A(s)}\left(L_{s}\right) .
$$

We first do the case where $m=2$. Suppose $C=A \cdot B$, and $L$ and $M$ are $r$-dimensional linear subspaces with $B(M) \subset L$. We must show that $D_{C^{*} C}(M)=$ $D_{A^{*} A}(L) \cdot D_{B^{*} B}(M)$. Let $u_{1}, \ldots, u_{r}$ be an orthonormal basis for $L$, and $v_{1}, \ldots, v_{r}$ an orthonormal basis for $M$. Write $B\left(v_{i}\right)=\sum x_{i j} u_{j}$, and let $X=\left(x_{i j}\right)$. Then

$$
\begin{aligned}
D_{C^{*} C}(M) & =\operatorname{det}\left(\left(B^{*} A^{*} A B v_{i}, v_{j}\right)\right)=\operatorname{det}\left(\left(A^{*} A B v_{i}, B v_{j}\right)\right) \\
& =\operatorname{det}(X) \cdot \operatorname{det}\left(\left(A^{*} A u_{i}, u_{j}\right)\right) \cdot \operatorname{det}\left(X^{*}\right)=\operatorname{det}\left(X X^{*}\right) \cdot \operatorname{det}\left(\left(A u_{i}, A u_{j}\right)\right) \\
& =\operatorname{det}\left(X X^{*}\right) \cdot D_{A^{*} A}(L) .
\end{aligned}
$$

But $D_{B^{*} B}(M)=\operatorname{det}\left(\left(B v_{i}, B v_{j}\right)\right)=\operatorname{det}\left(\sum x_{i k} \overline{x_{j l}}\left(u_{k}, u_{l}\right)\right)=\operatorname{det}\left(X X^{*}\right)$, which proves the claim. The general case is proved from this by induction, setting $A=A(1) \cdot \ldots \cdot A(m-1), B=A(m), M=L_{m}$, and $L=L_{m-1}$.

Theorem 19. Let $A(1), \ldots, A(m)$ be $n$ by $n$ matrices such that $A(1) \cdots A(m)=1$. Let $a_{1}(s) \geq \ldots \geq a_{n}(s)>0$ be the singular values of $A(s), 1 \leq s \leq m$. Suppose $I(1), \ldots, I(m)$ are subsets of cardinality $r$ of $\{1, \ldots, n\}$ such that the product $\prod \sigma_{I(s)}$ of the corresponding Schubert classes is not zero. Then

$$
\prod_{s=1}^{m} \prod_{i \in I(s)} a_{i}(s) \leq 1
$$

Note that $a_{1}(s)^{2} \geq \ldots \geq a_{n}(s)^{2}$ are the eigenvalues of $A(s)^{*} A(s)$. To prove the theorem, take flags $F$. $(s)$ corresponding to orthonormal bases of eigenvectors for $A(s)$. By Proposition 15, $D_{A(s)^{*} A(s)}\left(L_{s}\right) \geq \prod_{i \in I(s)} a_{i}(s)^{2}$ if $L_{s}$ is in $\Omega_{I(s)}\left(F_{\bullet}(s)\right)$. By the hypothesis on the nonvanishing of the product of Schubert classes, we may find a subspace $L$ in the intersection

$$
\Omega_{I(m)}\left(F_{\bullet}(m)\right) \cap \bigcap_{s=1}^{m-1} \Omega_{I(s)}(A(s+1) \cdot \ldots \cdot A(m))^{-1}\left(F_{\bullet}(s)\right) .
$$

Set $L_{s}=A(s+1) \cdot \ldots \cdot A(m)(L)$ for $1 \leq s<m$. Then $A(s)\left(L_{s}\right) \subset L_{s-1}$, and $L_{s}$ is in $\Omega_{I(s)}\left(F_{\bullet}(s)\right)$. By the two preceding propositions,

$$
1=D_{1}(L)=\prod_{s=1}^{m} D_{A(s) * A(s)}\left(L_{s}\right) \geq \prod_{s=1}^{m} \prod_{i \in I(s)} a_{i}(s)^{2} .
$$

Corollary. Let $A(1), \ldots, A(m)$ be $n$ by $n$ matrices, and let $C=A(1) \cdot \ldots \cdot A(m)$. Let $a(s)$ be the singular values of $A(s)$, and $c$ the singular values of $C$. Then for any $I(1), \ldots, I(m)$ and $K$ subsets of $\{1, \ldots, n\}$ of cardinality $r$, such that $\sigma_{\lambda(K)}$ appears in $\prod_{s=1}^{m} \sigma_{\lambda(I(s))}$,

$$
\prod_{k \in K} c_{k} \leq \prod_{s=1}^{m} \prod_{i \in I(s)} a_{i}(s)
$$


The proof is similar to that used in the additive case for eigenvalues: By a continuity argument, as in the proof of Proposition 7 , it suffices to consider the case where all the matrices are invertible. If $a_{1} \geq \ldots \geq a_{n}>0$ are the singular values of $A$, then $1 / a_{n} \geq \ldots \geq 1 / a_{1}$ are the singular values of $A^{-1}$. Apply the theorem to $C \cdot A(m)^{-1} \cdot \ldots \cdot A(1)^{-1}=1$, using the sets $K$ and $I(m)^{\prime}, \ldots, I(1)^{\prime}$, where $I^{\prime}$ denotes $\{n+1-i \mid i \in I\}$, exactly as in the proof of Theorem 17 .

In another remarkable paper [K12, Klyachko has recently proved the converse, and the corollary itself, which specializes to Theorem 16 for $m=2$. He deduces this from a computation of probability densities for random walks on the Lie algebra $\mathfrak{s u}(n)$ and the homogeneous space $S L(n, \mathbb{C}) / S U(n)$. Such densities for Euclidean 3 -space were computed by Lord Rayleigh.

10.7. Real symmetric and Hermitian quaternionic matrices. We prove the general versions of Theorems 3 and 4, Proposition 7, and Theorem 17 for real symmetric and Hermitian (self-conjugate) quaternionic matrices.

Theorem 20. The eigenvalues $\alpha(1), \ldots, \alpha(m)$, and $\gamma$ that occur for complex Hermitian matrices $A(1), \ldots, A(m)$ and $C=A(1)+\ldots+A(m)$ are the same as the eigenvalues that occur for real symmetric or for Hermitian quaternionic matrices.

For real symmetric matrices, it suffices to show that Proposition 7 is true for real symmetric matrices. The argument is similar to the complex case, using the fact that if $V$ is the complexification of a real vector space, then flags that are complexifications of real flags are Zariski dense in all flags. From this it follows that the Hermitian matrices constructed in Proposition 7 can all be taken to be real symmetric matrices. (See [Fu3] for details.)

A Hermitian quaternionic matrix is an $n$ by $n$ matrix $\mathrm{A}$ with entries in the quaternions $\mathbb{H}$ which is equal to the transpose of its conjugate. Such a matrix acts on the left on the (right) $\mathbb{H}$-space $\mathbb{H}^{n}$ of column vectors. Such a matrix again has all its eigenvalues real, and $\mathbb{H}^{n}$ has an orthonormal basis of eigenvectors. In this case, one need only prove that all the conditions $\left(*_{\mathcal{I}}\right)$ are necessary. This follows exactly as in the proofs of Propositions 2 and 7, together with the observation that Schubert calculus for the quaternionic Grassmannian $G r\left(r, \mathbb{H}^{n}\right)$ of (right) subspaces of $\mathbb{H}^{n}$ is exactly the same (but with all degrees doubled) as that for complex Grassmannians $\operatorname{Gr}\left(r, \mathbb{C}^{n}\right)$. This is proved in $[\mathrm{PR}]$.

Steinberg asks if one can characterize the eigenvalues of real skew-symmetric matrices $A, B$, and $C=A+B$. For any real matrix $A$, the matrix $\left(\begin{array}{cc}0 & A \\ -A^{t} & 0\end{array}\right)$ is skew-symmetric, so this may be related to the problem of characterizing singular values of real matrices $A, B$, and $C=A+B$.

\section{FinAl REMARKS}

It would be interesting to find more direct relations between the subjects addressed here that would give better explanations of why the questions in each subject have the same answers. It follows from the main theorems in this article that in each of these subjects the question of which triples $(\alpha, \beta, \gamma)$ occur for a given $n$ is completely determined by knowing the answer to the same question for all $r<n$. It is a challenge to find direct explanations for this in any of these situations. For example, is there a representation-theoretic explanation of the fact that knowing which irreducible representations of $G L_{n}(\mathbb{C})$ occur in tensor products is determined by the answer to this same question for $G L_{r}(\mathbb{C})$ for $r<n$ ? A similar 
question can be asked about the Schubert calculus for $\operatorname{Gr}\left(n, \mathbb{C}^{n+q}\right)$ being determined by the Schubert calculus for all $G r\left(r, \mathbb{C}^{n}\right)$ for $r<n$ (at least the part about which Schubert classes appear in a product). One has a similar mystery for the invariant factors problems. For the eigenvalue problem, Theorem 5 and Proposition 8 at least produce some smaller matrices.

Agnihotri and Woodward [AW] and Belkale [Be have proved an analogue of the Klyachko theorem for unitary matrices. They characterize the possible eigenvalues of unitary matrices $A(1), \ldots, A(m)$ whose product is the identity. Instead of being controlled by Schubert calculus as in the Hermitian eigenvalue problem, it is controlled by quantum Schubert calculus. Although there are algorithms for computing in this quantum Schubert calculus, there are not yet true analogues of the Littlewood-Richardson rule, nor are there useful criteria for the nonvanishing of such products.

One would like to see good analogues of these theorems for other Lie groups. We know that at least the saturation problem must be modified, but it seems reasonable to hope for sharp analogues of many of the theorems, at least for the other classical groups. The best results along these lines so far have been achieved by use of moment maps and coadjoint orbits, cf. [AW], BS], [He, OS]. Knutson and Tau end their paper [KT] with a precise conjecture.

One of the relations that comes from this work is that between representations of the symmetric group and Hermitian eigenvalues. There is a deep relation, conjectured by Baik, Deift, and Johansson [BDJ], and proved by Okounkov [. Any permutation $w$ in $S_{n}$, if written as a sequence of its values $w_{1}, \ldots, w_{n}$, determines a partition $\lambda(w)$ of $n: \lambda_{1}(w)$ is the length of the longest increasing subsequence of $w, \lambda_{1}(w)+\lambda_{2}(w)$ is the maximum sum of the lengths of the two disjoint increasing subsequences, and so on. The fact is that the distribution of these $\lambda_{k}(w)$ is exactly the same as the distribution of the eigenvalues of a random Hermitian $n$ by $n$ matrix, suitably normalized, as $n$ goes to infinity. Does this have any connection with the theorems presented here?

It may be worth pointing out explicitly that although the problems solved in this story range over several areas of mathematics - including linear algebra, commutative algebra, representation theory, intersection theory, and combinatorics - none of the people involved in the recent success came to the problems from any of these fields. Klyachko came from studying vector bundles on toric varieties; Totaro from studying filtered vector spaces using geometric invariant theory; Knutson, Agnihotri and Woodward came from symplectic geometry; Tao from harmonic analysis; and Belkale from the study of local systems on Riemann surfaces. Klyachko's article [K11] discusses some other interesting topics, such as spaces of polygons and Hermitian-Einstein metrics, that are related to the eigenvalue problem.

\section{REFERENCES}

[AW] S. Agnihotri and C. Woodward, Eigenvalues of products of unitary matrices and quantum Schubert calculus, math.AG/9712013, Math. Res. Lett. 5 (1998), 817-836. MR 2000a:14066

[AM] A. R. Amir-Moéz, Extreme properties of eigenvalues of a Hermitian transformation and singular values of the sum and product of linear transformations, Duke Math. J. 23 (1956), 463-476. MR 18:105j

[BDJ] J. Baik, P. Deift, and K. Johansson, On the distribution of the length of the longest increasing subsequence of random permutations, math.CO/9901118, J. Amer. Math. Soc. 12 (1999), 1119-1178. CMP 99:15 
[Be] P. Belkale, Local systems on $\mathbb{P}^{1} \backslash S$ for $S$ a finite set, Ph.D. thesis, University of Chicago, 1999.

[BS] A. Berenstein and R. Sjamaar, Projections of coadjoint orbits and the Hilbert-Mumford criterion, to appear in J. Amer. Math. Soc., math.SG/9810125.

[BG] F. A. Berezin and I. M. Gel'fand, Some remarks on spherical functions on symmetric Riemannian manifolds, Amer. Math. Soc. Transl. 21 (1962), 193-238. MR 27:1910

[Bi] P. Biane, Free probability for probabilists, MSRI preprint 1998-040.

$[\mathrm{Bu}]$ A. Buch, The saturation conjecture (after A. Knutson and T. Tao), to appear in l'Enseignement Math., math.C0/9810180.

[C] D. Carlson, Inequalities relating the degrees of elementary divisors within a matrix, Simon Stevin 44 (1970), 3-10. MR 43:232

[DST] J. Day, W. So, and R. C. Thompson, The spectrum of a Hermitian matrix sum, Linear Algebra Appl. 280 (1998), 289-332. MR 99f:15009

[DW] H. Derksen and J. Weyman, Semi-invariants of quivers and saturation for LittlewoodRichardson coefficients, to appear in J. Amer. Math. Soc.

[D] J. Deruyts, Essai d'une théorie générale des formes algébriques, Mém. Soc. Roy. Sci. Liège 17 (1892), 1-156.

[DO] I. Dolgachev and D. Ortland, Point sets in projective spaces and theta functions, Astérisque 165 (1988). MR 90i:14009

[DRW] A. H. Dooley, J. Repka and N. J. Wildberger, Sums of adjoint orbits, Linear and Multilinear Algebra 36 (1993), 79-101. MR 95k:22025

[F] Ky Fan, On a theorem of Weyl concerning eigenvalues of linear transformations, Proc. Nat. Acad. Sci. USA 35 (1949), 652-655. MR 11:600e

[Fi] M. Fiedler, Bounds for the determinant of the sum of Hermitian matrices, Proc. Amer. Math. Soc. 30 (1971), 27-31. MR 44:4021

[Fr] S. Friedland, Extremal eigenvalue problems for convex sets of symmetric matrices and operators, Israel J. Math. 15 (1973), 311-331. MR 49:2796

[Fu1] W. Fulton, Intersection Theory, Springer-Verlag, 1984, 1998. MR 85k:14004

[Fu2] — Young Tableaux, Cambridge University Press, 1997. MR 99f:05119

[Fu3] _ Eigenvalues of sums of Hermitian matrices (after A. Klyachko), Séminaire Bourbaki 845, June, 1998, Astérisque 252 (1998), 255-269. CMP 99:13

[FH] W. Fulton and J. Harris, Representation Theory, Springer-Verlag, 1991. MR 93a:20069

[GN] I. M. Gel'fand and M. A. Naimark, The relation between the unitary representations of the complex unimodular group and its unitary subgroup, Izvestiya. Akad. Nauk SSSR. Ser. Mat 14 (1950), 239-260 (Russian). MR 12:9i

[Gr] D. Grayson, Reduction theory using semistability, Comm. Math. Helvetici 59 (1984), 600634. MR 86h:22018

[He] G. J. Heckman, Projections of orbits and asymptotic behavior of multiplicities for compact connected Lie groups, Invent. Math. 67 (1982), 333-356. MR 84d:22019

[HR] U. Helmke and J. Rosenthal, Eigenvalue inequalities and Schubert calculus, Math. Nachr. 171 (1995), 207- 225. MR 96b:15039

[HZ] J. Hersch and B. Zwahlen, Évaluations par défaut pour une summe quelconque de valeurs propres $\gamma_{k}$ d'un opérateur $C=A+B$, à l'aide de valeurs propres $\alpha_{i}$ de $A$ et $\beta_{j}$ de $B, \mathrm{C} . \mathrm{R}$. Acad. Sc. Paris 254 (1962), 1559-1561. MR 24:A2583

[H1] A. Horn, Doubly stochastic matrices and the diagonal of a rotation matrix, Amer. J. Math. 76 (1954), 620-630. MR 16:105c

[H2] _ Eigenvalues of sums of Hermitian matrices, Pacific J. Math. 12 (1962), 225-241. MR 25:3941

[Joh] C. R. Johnson, Precise intervals for specific eigenvalues of a product of a positive definite and a Hermitian matrix, Linear Algebra Appl. 117 (1989), 159-164.

[JS] C. R. Johnson and E. A. Schreiner, The relationship between $A B$ and BA, Amer. Math. Monthly 103 (1996), 578-582. MR 97e:15007

[J] S. Johnson, The Schubert calculus and eigenvalue inequalities for sums of Hermitian matrices, Ph.D. thesis, University of California. Santa Barbara, 1979.

[K] T. Klein, The multiplication of Schur-functions and extensions of p-modules, J. London Math. Society 43 (1968), 280-284. MR 37:4061

[Kle] S. L. Kleiman, The transversality of a general translate, Compositio Math. 28 (1974), 287297. MR 50:13063 
[K11] A. A. Klyachko, Stable bundles, representation theory and Hermitian operators, Selecta Math. 4 (1998), 419- 445. MR 2000b:14054

[Kl2] — Random walks on symmetric spaces and inequalities for matrix spectra, preprint, 1999.

[Kn] A. Knutson, The symplectic and algebraic geometry of Horn's problem, math.LA/9911088.

$[\mathrm{KT}]$ A. Knutson and T. Tao, The honeycomb model of $G L_{n}(\mathbb{C})$ tensor products $I$ : proof of the saturation conjecture, J. Amer. Math. Soc. 12 (1999), 1055-1090. MR 2000c:20066

[KTW] A. Knutson, T. Tao and C. Woodward, Honeycombs II: facets of the LittlewoodRichardson cone, to appear.

[Ko] B. Kostant, Lie algebra cohomology and the generalized Borel-Weil theorem, Annals of Math. 74 (1961), 329-387. MR 26:265

[La] S. Lang, Algebra, Second Edition, Addison-Wesley, 1984. MR 86j:00003

[Le] L. Lesieur, Les problèmes d'intersection sur une variété de Grassmann, C. R. Acad. Sci. 225 (1947), 916-917. MR 9:304c

[L1] B. V. Lidskii, The proper values of the sum and product of symmetric matrices, Dokl. Akad. Nauk SSSR 74 (1950), 769-772 (Russian).

[L2] B. V. Lidskii, Spectral polyhedron of the sum of two Hermitian matrices, Funct. Anal. Appl. 16 (1982), 139-140. MR 83k:15009

[LR] D. E. Littlewood and A. R. Richardson, Group characters and algebra, Philos. Trans. Roy. Soc. London A 233 (1934), 99-141.

[Mac] I. G. Macdonald, Symmetric Functions and Hall Polynomials, Second edition, Clarendon, Oxford, 1995. MR 96h:05207

[MFK] D. Mumford, J. Fogarty and F. Kirwan, Geometric Invariant Theory, Third Enlarged Edition, Springer-Verlag, 1994. MR 95m:14012

[O] A. Okounkov, Random matrices and random permutations, math.CO/9903176.

[OS] L. O'Shea and R. Sjamaar, Moment maps and Riemannian symmetric pairs, to appear in Math. Ann., math.SG/9902059.

[PR] P. Pragacz and J. Ratajski, Formulas for Lagrangian and orthogonal degeneracy loci: the $\widetilde{Q}$-polynomials approach, Compositio Math. 107 (1997), 11-87. MR 98g:14063

[QS] J. F. Queiró and E. Marques de Sá, Singular values and invariant factors of matrix sums and products, Linear Algebra Appl. 225 (1995), 43-56. MR 96e:15012

[R] R. C. Riddell, Minimax problems on Grassmann manifolds. Sums of eigenvalues, Adv. in Math. 54 (1984), 107-199. MR 86k:58019

[SQS] A. P. Santana, J. F. Queiró and E. Marques de Sá, Group representations and matrix spectral problems, Linear and Multilinear Algebra 46 (1999), 1-23. CMP 2000:02

[So] F. Sottile, The special Schubert calculus is real, Electron. Res. Announc. Amer. Math. Soc. 5 (1999), 35-39. MR 2000c:14074

[Ta] T. Y. Tam, A unified extension of two results of Ky Fan on the sum of matrices, Proc. Amer. Math. Soc. 126 (1998), 2607-2614. MR 98m:15032

[Thi] G. P. A. Thijsse, The local invariant factors of a product of holomorphic matrix functions: the order 4 case, Integral Equations Operator Theory 16 (1993), 277-304, 605. MR 94c:47021a; MR 94c:47021b

[Th1] R. C. Thompson, An inequality for invariant factors, Proc. Amer. Math. Soc. 86 (1982), 9-11. MR 83k:15014

[Th2] - Smith invariants of a product of integral matrices, Contemp. Math. 47 (1985), 401-435. MR 87k:15024

[Th3] — Invariant factors of algebraic combinations of matrices, Frequency domain and state space methods for linear systems, North Holland, 1986, pp. 73-87. MR 89c:15001

[TF] R. C. Thompson and L. Freede, On the eigenvalues of a sum of Hermitian matrices, Linear Algebra Appl. 4 (1971), 369-376. MR 44:5330

[TT1] R. C. Thompson and S. Therianos, The eigenvalues and singular values of matrix sums and products. VII, Canad. Math. Bull 16 (1973), 561-569. MR 50:4629

[TT2] _ On a construction of B. P. Zwahlen, Linear and Multilinear Algebra 1 (1973/74), 309-325. MR 49:5044

[T] B. Totaro, Tensor products of semistables are semistable, Geometry and Analysis on complex Manifolds, World Sci. Publ., 1994, pp. 242-250. MR 98k:14014

[W] H. Weyl, Das asymtotische Verteilungsgesetz der Eigenwerte lineare partieller Differentialgleichungen, Math. Ann. 71 (1912), 441-479. 
[Wi] H. Wielandt, An extremum property of sums of eigenvalues, Proc. Amer. Math. Soc. 6 (1955), 106-110. MR 16:785a

[X] B. Y. Xi, A general form of the minimum-maximum theorem for eigenvalues of self-conjugate quaternion matrices, Nei Mongol Daxue Xuebao Ziran Kexue 22 (1991), 455-458 (Chinese). MR 92g:15025

[Z] A. Zelevinsky, Littlewood-Richardson semigroups, math.CO/9704228, New Perspectives in Algebraic Combinatorics (L. J. Billera, A. Björner, C. Greene, R. E. Simion, R. P. Stanley, eds.), Cambridge University Press (MSRI Publication), 1999, pp. 337-345.

[Zw] B. P. Zwahlen, Über die Eigenwerte der Summe zweier selbstadjungierter Operaten, Comm. Math. Helv. 40 (1965), 81-116. MR 32:7566

University of Michigan, Ann Arbor, MI 48109-1109

E-mail address: wfulton@math.lsa.umich.edu 